\title{
Cannabidiol Counteracts the Psychotropic Side-Effects of $\Delta$-9-Tetrahydrocannabinol in the Ventral Hippocampus through Bidirectional Control of ERK1-2 Phosphorylation
}

\author{
- Roger Hudson, ${ }^{1,2}$ Justine Renard, ${ }^{1,2}$ Christopher Norris, ${ }^{1,2}$ Walter J. Rushlow, ${ }^{1,2,3}$ and Steven R. Laviolette ${ }^{1,2,3}$ \\ ${ }^{1}$ Addiction Research Group, ${ }^{2}$ Department of Anatomy and Cell Biology, and ${ }^{3}$ Department of Psychiatry, Schulich School of Medicine and Dentistry, \\ University of Western Ontario, London, Ontario, Canada N6A 3K7
}

Evidence suggests that the phytocannabinoids $\Delta$-9-tetrahydrocannabinol (THC) and cannabidiol (CBD) differentially regulate salience attribution and psychiatric risk. The ventral hippocampus (vHipp) relays emotional salience via control of dopamine (DA) neuronal activity states, which are dysregulated in psychosis and schizophrenia. Using in vivo electrophysiology in male Sprague Dawley rats, we demonstrate that intra-vHipp THC strongly increases ventral tegmental area (VTA) DA neuronal frequency and bursting rates, decreases GABA frequency, and amplifies VTA beta, gamma and $\varepsilon$ oscillatory magnitudes via modulation of local extracellular signal-regulated kinase phosphorylation (pERK1-2). Remarkably, whereas intra-vHipp THC also potentiates salience attribution in morphine placepreference and fear conditioning assays, CBD coadministration reverses these changes by downregulating pERK1-2 signaling, as pharmacological reactivation of pERK1-2 blocked the inhibitory properties of CBD. These results identify vHipp pERK1-2 signaling as a critical neural nexus point mediating THC-induced affective disturbances and suggest a potential mechanism by which CBD may counteract the psychotomimetic and psychotropic side effects of THC.

Key words: cannabidiol; delta-9-tetrahydrocannabinol; dopamine; extracellular signal-regulated kinase; ventral hippocampus; ventral tegmental area

\section{Significance Statement}

Strains of marijuana with high levels of delta-9-tetrahydrocannabinol (THC) and low levels of cannabidiol (CBD) have been shown to underlie neuropsychiatric risks associated with high-potency cannabis use. However, the mechanisms by which CBD mitigates the side effects of THC have not been identified. We demonstrate that THC induces cognitive and affective abnormalities resembling neuropsychiatric symptoms directly in the hippocampus, while dysregulating dopamine activity states and amplifying oscillatory frequencies in the ventral tegmental area via modulation of the extracellular signal-regulated kinase (ERK) signaling pathway. In contrast, CBD coadministration blocked THC-induced ERK phosphorylation, and prevented THC-induced behavioral and neural abnormalities. These findings identify a novel molecular mechanism that may account for how CBD functionally mitigates the neuropsychiatric side effects of THC.

\section{Introduction}

Chronic or acute use of high-potency cannabis is associated with numerous neuropsychiatric side effects, including dysregulation of emotional processing and associative memory formation (Di

Received March 28, 2019; revised Aug. 16, 2019; accepted Sept. 3, 2019.

Author contributions: R.H., W.J.R., and S.R.L. designed research; R.H., J.R., and C.N. performed research; R.H., J.R., C.N., and W.J.R. analyzed data; R.H. wrote the paper; W.J.R. and S.R.L. edited the paper.

This work was supported by the Canadian Institutes of Health Research (CIHR), the National Science and Engineering Research Council of Canada (NSERC), Mitacs, and by a Vanier Canada Graduate Scholarship awarded to R.H. The authors declare no competing financial interests.

Correspondence should be addressed to Steven R. Laviolette at steven.laviolette@schulich.uwo.ca.

https://doi.org/10.1523/JNEUROSCI.0708-19.2019

Copyright $\odot 2019$ the authors
Forti et al., 2009). However, given the phytochemical complexity of cannabis, increasing evidence suggests highly divergent psychotropic effects after exposure to distinct cannabis constituents. Thus, while the neuropsychiatric side effects of cannabis are associated with $\Delta$-9-tetrahydrocannabinol (THC), clinical and preclinical findings demonstrate that cannabidiol (CBD), the major nonpsychoactive phytocannabinoid in cannabis, possesses antipsychotic and anxiolytic properties (Zuardi et al., 2006; Leweke et al., 2012). Indeed, whereas THC induces dysregulation of subcortical dopamine (DA) transmission and affective salience processing (Bhattacharyya et al., 2012; Palaniyappan et al., 2013; Renard et al., 2017a,b), CBD normalizes aberrant DA signaling and regulates affective stimulus processing (Norris et al., 2016; Renard et 
al., 2016). Nevertheless, the precise neurobiological mechanisms by which CBD may mitigate the neuropsychiatric side effects of THC are not understood.

The ventral hippocampus (vHipp) is an integrative structure subserving mesocorticolimbic DA signaling and emotional processing and is implicated in schizophrenia-related neuropathology (Grace, 2010). For example, disinhibition of the vHipp distorts emotional salience and induces a schizophrenia-like phenotype by dysregulating activity of ventral tegmental area (VTA) DA neurons and mesolimbic DA transmission (Legault et al., 2000; Floresco et al., 2001; Loureiro et al., 2015). vHipp morphometric abnormalities and dysregulated connectivity between the vHipp and frontotemporal structures are pathological features of schizophrenia (Szeszko et al., 2003; Meyer-Lindenberg et al., 2005). Importantly, use of THC-rich cannabis induces vHipp abnormalities and learning and memory disturbances that are mitigated by CBD (Bhattacharyya et al., 2010, 2015; Englund et al., 2013; Beale et al., 2018). Thus, pathological vHipp overdrive may represent a convergent link contributing to disturbances in affective salience processing and memory function observed in schizophrenia and after THC exposure (Laviolette and Grace, 2006).

CBD mitigates psychosis-like neuronal, behavioral, and molecular endophenotypes associated with schizophrenia, for example, by inhibiting amphetamine and MK-801-induced dysregulation of DA transmission, and associated beta $(\beta)$ and gamma $(\gamma)$ oscillatory patterns in reverse-translational schizophrenia models (Benes et al., 2007; Gomes et al., 2015; Moreira and Guimarães, 2005; Renard et al., 2016, 2017a). Furthermore, CBD counteracts several signal transduction cascades associated with THC-induced impairments, including the hippocampal mitogen-activated protein kinase/extracellular signal-regulated protein kinase (ERK) pathway (Derkinderen et al., 2003; Solinas et al., 2013; Elbaz et al., 2015). Considering that vHipp dysfunction is common to THC-induced neuropsychiatric impairments and schizophrenia, one possibility is that the antipsychotic properties of CBD are attributable to competing actions by CBD on local vHipp function.

In the present study, we investigated the hypothesis that intravHipp THC and CBD differentially impact emotional memory processing and VTA neural activity via distinct actions within the vHipp ERK signaling pathway. To elucidate the specificity of THC on the vHipp ERK signal transduction cascade, we coadministered THC alongside the highly selective and potent MEK1-2 inhibitor, U0126, which functionally antagonizes AP-1 transcription via MEK inhibition (Duncia et al., 1998). Additionally, to characterize the properties of combined THC $+\mathrm{CBD}$ on ERK activity, we coadministered THC + CBD alongside eicosapentaenoic acid (EPA), which promotes ERK phosphorylation via interaction with Thr235 and increases in upstream ratelimiting H-Ras protein expression levels. We report that intravHipp THC potentiates the affective salience of normally nonsalient rewarding and aversive memory cues, while increasing VTA DA neuronal activity states, decreasing VTA GABAergic activity, and concomitantly amplifying $\beta, \gamma$, and $\varepsilon$ oscillatory frequencies within the VTA via an ERK-dependent mechanism. Remarkably, CBD coadministration reversed THC-induced changes in VTA neural activity and emotional memory processing via downregulation of vHipp ERK phosphorylation states. These findings identify for the first time a precise molecular and neuronal mechanism by which CBD may mitigate the neuropsychiatric side effects of THC directly in the vHipp, a brain region known to be pathologically impacted by cannabis exposure and schizophrenia.

\section{Materials and Methods}

Subjects. Male Sprague Dawley rats (Charles River Laboratories) weighing 250-300 g at the start of experiments were single-housed under controlled conditions $\left(22-23^{\circ} \mathrm{C}\right.$, lights on at 07:00, lights off at 19:00) with ad libitum access to food and water. All experimental protocols were approved by the Animal Care and Veterinary Services Committee at Western University and were performed in accordance with recommendations provided by the Canadian Council on Animal Care.

Surgical procedures. Rats were anesthetized with a 2:1 mixture of ketamine $(100 \mathrm{mg} / \mathrm{ml}$; Narketan $)$ and xylazine $(20 \mathrm{mg} / \mathrm{ml}$; Bayer $)$ and placed in a stereotaxic device. Stainless steel guide cannulae (22 gauge; Plastics One) were implanted bilaterally into the vHipp at the following coordinates: AP: $-5.6 \mathrm{~mm}$ from bregma, LM: $\pm 5.0 \mathrm{~mm}, \mathrm{DV}:-6.8 \mathrm{~mm}$ from the dural surface. Guide cannulae were secured in place using jeweler's screws and dental acrylic cement. To minimize pain and inflammation, meloxicam ( $1 \mathrm{mg} / \mathrm{kg}$, s.c.; Boehringer Ingelheim) was administered before surgeries and on the initial postoperative day. Behavioral testing began 1 week after recovery. After completion of behavioral experiments, rats received an overdose of sodium pentobarbital $(240 \mathrm{mg} / \mathrm{kg}$, i.p., Euthanyl) and brains were extracted and postfixed $24 \mathrm{~h}$ in $10 \%$ formalin before being placed in a $25 \%$ formalin-sucrose solution for 1 week. Brains were sliced $(60 \mu \mathrm{m})$ using a cryostat and stained with cresyl violet. Injector tip placements were localized using a light microscope. Rats with cannula placements found outside the anatomical boundaries of the vHipp (as defined by Paxinos and Watson, 2007) were excluded from data analysis ( $n=7$ total). An additional eight rats were removed from subsequent experiments after blockade of cannulae throughout the series of experiments.

Drug administration. The following drugs were used during behavioral or electrophysiological experiments: THC (Cayman Chemical), CBD (Tocris Bioscience), the selective MEK1/MEK2 inhibitor U0126 (Tocris Bioscience), the $\omega$-3 fatty acid eicosapentaenoic acid (EPA; Tocris Bioscience), sucrose (Sigma-Aldrich), and morphine sulfate (JohnsonMatthey). THC and EPA in ethanol were each dissolved in cremophor and saline (1:1:18). Nitrogen gas was used to evaporate ethanol from the final THC and EPA solutions. CBD was dissolved in cremophor and saline (1:18). U0126 was dissolved in DMSO and then diluted in sterile saline to achieve a $25 \%$ DMSO concentration. Morphine sulfate was dissolved in physiological saline, with $\mathrm{pH}$ adjusted to 7.4. A solution of cremophor and saline (1:18) was infused as a Vehicle control.

Intra-vHipp microinfusions were performed immediately before each behavioral assay or conditioning session. A total volume of $0.5 \mathrm{ml}$ per hemisphere was delivered via 28 -gauge microinfusion injectors over 1 $\mathrm{min}$. To ensure adequate drug diffusion, microinjectors were left in place for an additional 1 min after drug infusion.

Protein expression analyses. To evaluate the local effects of intra-vHipp phytocannabinoids on expression of pERK, ERK, and the ratio of pERK: ERK, a subset of rats received bilateral intra-vHipp microinfusions of vehicle (VEH), THC (100 ng), CBD (100 ng), THC+CBD (100 ng + 100 $\mathrm{ng}), \mathrm{THC}+\mathrm{U} 0126(100 \mathrm{ng}+1 \mu \mathrm{g})$ or THC $+\mathrm{CBD}+\mathrm{EPA}(100 \mathrm{ng}+100$ $\mathrm{ng}+1 \mathrm{~mm}$ ) $5 \mathrm{~min}$ before being euthanized. Brains were rapidly removed, and flash frozen at $-80^{\circ} \mathrm{C}$. Coronal sections $(95 \mu \mathrm{m})$ containing the vHipp were cut on a cryostat and slide mounted. Bilateral microdissections surrounding the injector sites were obtained $(\sim 2.5 \mathrm{mg}$ total tissue per subject), using light microscopy to identify and avoid any regions with reactive gliosis. The Western blotting procedure was performed as described previously (Lyons et al., 2013) using $\sim 12.5 \mu \mathrm{g}$ of collected tissue per blot. Primary antibody dilutions were as follows: $\alpha$-tubulin (1:1,000,000; Sigma-Aldrich), pERK (1: 1,000; Cell Signaling Technology, and ERK (1: 2000; Cell Signaling Technology). Species-appropriate horseradish peroxidase (HRP)-conjugated secondary antibodies (LICOR IRDye 680RD and IRDye 800CW; Thermo Scientific) were each used at a dilution of 1: 10,000. Densitometry measurements were obtained using LI-COR Odyssey digital imaging software and Image Studio analysis software by normalizing the intensity of each sample's target protein band to the respective $\alpha$-tubulin intensity. 
VTA neuronal activity recordings and analysis. Extracellular single-unit electrophysiological recordings were performed in vivo in adult rats weighing 300-450 g. The recordings were taken either from putative dopaminergic (DA) VTA neurons or from non-DA GABAergic VTA neurons. In vivo extracellular single-unit recordings in the VTA were performed as described previously (Loureiro et al., 2015). Briefly, rats were anesthetized with urethane (1.5 g/kg, i.p.; Sigma-Aldrich) and placed in a stereotaxic frame with body temperature maintained at $37^{\circ} \mathrm{C}$. A scalp incision was made, and holes were drilled in the skull overlaying the VTA and vHipp. For intra-vHipp microinfusions, a $10 \mu$ l Hamilton syringe was slowly lowered into the vHipp using the stereotaxic coordinates described above. For intra-VTA recordings, glass microelectrodes (average impedance of $6-8 \mathrm{M} \Omega$ ) filled with a $2 \mathrm{M}$ sodium acetate solution containing $2 \%$ pontamine sky blue were lowered with a hydraulic micropositioner (Kopf Instruments 640) to the following coordinates: AP: $-5.0 \mathrm{~mm}$ from bregma, $\mathrm{L}: \pm 0.7 \mathrm{~mm}, \mathrm{DV}:-7.0$ to $-9.0 \mathrm{~mm}$ from the dural surface. A bone screw was placed over the cerebellum and connected with the return of the headstage, serving as a reference electrode. The electrophysiological properties of spontaneously active neurons were sampled by vertically passing the electrode through the VTA cell body region. A minimum washout duration of $1 \mathrm{~h}$ was allowed between intra-vHipp infusions. Vertical tracks were made in a predefined pattern, with each track separated by $200 \mu \mathrm{m}$.

Extracellular signals were amplified (x5000) using a MultiClamp700B amplifier (Molecular Devices), digitized at $25 \mathrm{kHz}$ and recorded through a Digidata1440A acquisition system (Molecular Devices) and pClamp10 software. Wide-band VTA signal recordings were fed through two channels of the digitizer and filtered to obtain single unit recordings (bandpass between 0.3 and $3 \mathrm{kHz}$ ) and local field potentials (LFPs; low pass at $0.3 \mathrm{kHz}$ ). VTA DA neurons were identified according to well established electrophysiological features (Grace and Bunney, 1983): (1) a relatively long action potential width $(>2.5 \mathrm{~ms}),(2)$ a slow, spontaneous firing rate (2-9 Hz), (3) a triphasic waveform consisting of a notch on the rising phase followed by a delayed after potential, and (4) a single irregular or bursting firing pattern. In contrast, VTA non-DAergic cells were characterized based upon: (1) a narrow action potential width $(<1 \mathrm{~ms}),(2)$ a biphasic waveform, (3) relatively fast firing rates $(>10 \mathrm{~Hz})$, and (4) the absence of burst firing.

Electrophysiological analyses were performed using the Clampfit10 (Molecular Devices) software package. Firing frequencies of isolated VTA neurons after intra-vHipp microinfusions were normalized to the respective baseline firing frequency. The response patterns of VTA neurons to vHipp microinfusions were determined by comparing the neuronal frequency rates between the 5 min preinfusion versus postinfusion recording epochs. The 5 min preinfusion epoch was commenced after at least 2 min of stable physiological (single unit and LFP) activity. The 5 min postinfusion epoch commenced immediately after the 1 min intravHipp infusion. We ensured minimum $1 \mathrm{~h}$ delay between recordings to prevent effects infusions, and recordings within each animal were performed within 1 drug condition. Classification of drug infusion effects used a criterion of $>10 \%$ change in firing frequency relative to preinfusion to be classified as an "increase" or "decrease" effect. Neurons displaying $<10 \%$ change in firing frequency parameters after infusion were classified as 'no change'. In VTA DA neurons, we also analyzed the proportion of action potentials firing in the phasic bursting modality. The onset of a burst event was defined as the occurrence of two or more consecutive spikes within an interspike interval of $<80 \mathrm{~ms}$.

LFP signals were analyzed using NeuroExplorer (Nex Technologies). Recordings were first decimated to $1 \mathrm{kHz}$, and lowpass filtered (IIR Butterworth filter at $170 \mathrm{~Hz}$; filter order set to 3). A spectrogram function was then used to calculate the power of oscillations at frequencies between $0-100 \mathrm{~Hz}$ (window length $2 \mathrm{~s}$; shift $0.5 \mathrm{~s}$ ). Preinfusion and postinfusion parameters remained the same as outlined previously. Power values for a given frequency were averaged over the recording epoch and normalized so that the sum of all power spectrum values equals 1 . The total power was calculated by adding the power values at frequencies between $0-59$ and $61-100 \mathrm{~Hz}$, and power values at $60 \pm 1 \mathrm{~Hz}$ were excluded from all calculations. Neural oscillations were defined within specific frequency bands as follows: delta band, $0-4 \mathrm{~Hz}$; theta band, $4-7$
$\mathrm{Hz}$; alpha band, $7-14 \mathrm{~Hz} ; \beta$ band, $14-30 \mathrm{~Hz} ; \gamma$ band, $30-80 \mathrm{~Hz}$; and $\varepsilon$ band, 90-100 Hz. Every recording was taken at a different electrode location throughout VTA.

Positions of recording electrodes were marked with an iontophoretic deposit of pontamine sky blue dye ( $-19.5 \mathrm{~mA}$, continuous current for 15 $\mathrm{min}$ ) for histological analyses, which were performed as described previously (Renard et al., 2017a,b). Recordings obtained from two rats were excluded from electrophysiological data analysis because histological analysis revealed iontophoretic deposits beyond the anatomical boundaries of the VTA as defined by Paxinos and Watson (2007).

Behavioral testing. A battery of behavioral assays was conducted to examine the effects of acute intra-vHipp THC vs CBD infusions on affective and anxiety-related behaviors. Between experiments, rats were returned to the home cage for a minimum of $3 \mathrm{~d}$. Rats were randomly assigned across experiments and counterbalanced within groups. Separate cohorts of rats received the conditioned place preference, contextdependent, or context-independent fear conditioning procedures as final tests, as these (opiate exposure and high stress) tests were considered terminal. We performed the following ordered series of behavioral assays:

Open-field test. Rats were placed in an automated open field activity chamber (Med Associates) for $30 \mathrm{~min}$. Total distance moved, time spent inactive in the center compartment of the chamber (initial 5 min only), and stereotypy times were recorded and analyzed. Time spent inactive in the center compartment relative to the outer compartment were measured as an index of anxiety as higher states of anxiety are associated with lower exploration times in the central regions of the open field chamber and greater times spent in the peripheral zone (Ohl, 2003). Stereotypy was defined as time spent mobile within a software-generated, predefined zone in the open field chamber. At time 0 , the program centers this predefined zone on the subject. When the subject moves outside this zone, the activity is considered ambulatory and the zone recenters on the subject. If the subject remains inside the recentered zone for longer than a software-defined resting delay (2500 ms), the subject's activity is considered stereotypic until they move outside the zone.

Light/dark box. This test exploits a rat's natural aversion to bright environments and attributes greater time spent in an illuminated environment as reflecting lower anxiety levels. The test was performed as previously described (Renard et al., 2017a). At the start of the experiment, a rat was placed in the center of the lighted box with its head facing the wall opposite the door and allowed to freely explore both compartments for $8 \mathrm{~min}$. A zone entry was considered to have begun when the rat placed all 4 paws in that zone. Experiments were videotaped with an automated video-tracking system (ANY-maze; Stoelting). Behaviors analyzed included percentage time in light side, and latency to second transition (latency time to exit the dark box and reenter the light box), which are each robust indicators of anxiety-like behavior and sensitive to both anxiogenic and anxiolytic treatments ${ }^{62}$.

Sucrose preference test. To allow for acclimation to a palatable liquidsucrose solution, rats received $48 \mathrm{~h}$ ad libitum access to $2 \%$ sucrose solution in bottles suspended in their home cages, without access to water. After acclimation, rats were deprived of water and $2 \%$ sucrose solution for $12 \mathrm{~h}$ before testing. During the $1 \mathrm{~h}$ test, rats were given ad libitum access to two bottles, one containing water and one containing $2 \%$ sucrose solution. Fluid volumes consumed during testing were normalized according to body weight and sucrose preference was calculated and expressed as percentage of total fluid intake.

Context-dependent and context-independent fear conditioning. To study the effects of intra-vHipp phytocannabinoids on aversive memory processing, rats were tested in context-dependent or context-independent olfactory cue fear conditioning procedures as described previously (Loureiro et al., 2016). During context-dependent fear conditioning, associative contextual stimuli of the context (black and white stripes, or polka dots) were paired through counterbalancing with subthreshold levels of foot shock ( 10 shocks, $1 \mathrm{~s}, 0.4 \mathrm{~mA}$, administered at random time intervals during a single $20 \mathrm{~min}$ conditioning session) that we have previously shown do not produce significant fear memories (Loureiro et al., 2016). Twenty-four hours after conditioning, subjects were reexposed for 10 min to the conditioning context for testing, in a drug-free state. For the 
context-independent fear conditioning protocol, subjects were habituated for $30 \mathrm{~min}$ to the 'safe' context $24 \mathrm{~h}$ before conditioning. Two odors were used during context-independent olfactory fear conditioning in the 'shock' context - almond and peppermint fragrance oils. One odor was paired with a foot shock (conditioned stimulus $\left(\mathrm{CS}^{+}\right.$), and the other paired with the absence of foot shock $\left(\mathrm{CS}^{-}\right)$in a counterbalanced fashion. $10 \mathrm{CS}^{+}$and $10 \mathrm{CS}^{-}$pairings were presented during the $20 \mathrm{~min}$ conditioning session. Twenty-four hours after conditioning, subjects were reexposed for $5 \mathrm{~min}$ to each CS in a counterbalanced style for testing, in a drug-free state. Time freezing was recorded over $10 \mathrm{~min}$ using automated video tracking (ANY-maze).

Conditioned place preference. We used a conditioned place preference (CPP) procedure, as previously described (Lintas et al., 2011). Briefly, subjects were habituated to a neutral gray context for $20 \mathrm{~min}$ the day before conditioning onset. For conditioning days, saline or morphine injections (i.p.) were paired with one of two environments that differed in color (black vs white), texture (Plexiglas floor vs bedding), and smell ( $0.4 \mathrm{ml}$ of $2 \%$ acetic acid vs woodchips scent). For CPP testing, rats are given the free choice to spend time in either of the previously experienced (Drug vs Veh) conditioning environments, which are separated by a narrow $(10 \mathrm{~cm})$ gray strip of Plexiglas. As reported previously, rats display no baseline preference for either of these two environments (Laviolette and van der Kooy, 2003). Rats received four morphine-environment and four saline-environment conditioning sessions ( 1 session per day on alternating days, $8 \mathrm{~d}$ conditioning in total) in a fully counterbalanced design. We used a subreward threshold conditioning dose of morphine ( $0.05 \mathrm{mg} / \mathrm{kg}$ i.p. $)$ that we have previously demonstrated to produce no significant CPP (Lintas et al., 2011). Rats received intraperitoneal injections immediately before being placed in saline- or morphine-paired environments for conditioning sessions lasting $30 \mathrm{~min}$ each. Intracranial microinfusions directly preceded intraperitoneal injections and were given before all conditioning trials. Two days after conditioning, rats were tested (drug-free) for place preference during a $10 \mathrm{~min}$ test, and times spent in each environment were recorded with an automated video-tracking system (ANY-maze). CPP behavior is expressed as percentage time spent in morphine and saline-paired environments.

Statistical analyses. The data were analyzed using one or two-way analyses of variance (ANOVA) followed by Newman-Keul's (electrophysiological data) or Fisher's LSD tests (molecular and behavioral analyses) for multiple comparisons (alpha $<0.05$ ). When appropriate, planned comparisons were performed using $t$ tests, as indicated. All analyses were performed using Sigmaplot (version 12.0 for Windows), and only the exact values of significant analyses are reported.

\section{Results \\ THC and CBD differentially regulate local ERK1-2 phosphorylation states in the vHipp}

At the molecular level, THC and CBD differentially modulate cellular ERK1-2 signaling, with THC activating and CBD inhibiting ERK1-2 phosphorylation (Derkinderen et al., 2003; Elbaz et al., 2015). Accordingly, we first examined whether intra-vHipp vehicle (VEH; $n=6)$, THC (100 ng/0.5 $\mu \mathrm{l} ; n=6)$, CBD (100 $\mathrm{ng} / 0.5 \mu \mathrm{l} ; n=6)$, or a combination of THC (100 ng) + CBD (100 ng/0.5 $\mu \mathrm{l} ; n=6)$ may differentially modulate local vHipp ERK1-2 phosphorylation (pERK1-2) states. To characterize the ability of THC to increase pERK1-2 expression directly in the vHipp we coadministered THC alongside the MEK1-2 inhibitor, U0126 ( $1 \mu \mathrm{g} ; n=5)$, at a dose previously characterized as having pharmacologically specific actions on neuronal ERK1-2 expression levels (Lyons et al., 2013). In addition, to characterize the actions of THC $+\mathrm{CBD}$ on pERK1-2 expression levels, we coadministered the THC+CBD with the ERK-activating agent eicosapentaenoic acid (EPA; $1 \mathrm{~mm} ; n=5$ ), at a dose previously described to elevate pERK1-2 phosphorylation (Maher et al., 2004; Salvati et al., 2008).

Mixed-measures ANOVA revealed a significant main effect of group $\left(F_{(5,28)}=10.18, p<0.001\right)$, but no significant effect of isoform factor $\left(F_{(1,28)}=0.328, p>0.05\right)$, or interaction between group and isoform factors $\left(F_{(5,28)}=0.297, p>0.05\right)$. Post hoc comparisons using Fisher's LSD on marginal means revealed that THC significantly increased levels of vHipp pERK1-2 expression relative to VEH $(p=0.001), \mathrm{CBD}(p<0.001), \mathrm{THC}+\mathrm{CBD}(p<$ $0.001)$, and THC $+\mathrm{U} 0126$ groups $(p=0.04)$. Thus, intra-vHipp THC strongly increases local pERK1-2 expression via upstream MEK1-2 signaling. In contrast, whereas $\mathrm{CBD}$ has no significant effects relative to VEH, the THC $+\mathrm{CBD}$ combination blocks the effects of THC and significantly downregulates pERK1-2 expression relative to VEH $(p=0.004)$. Furthermore, the THC $+\mathrm{CBD}$ decrease of vHipp pERK1-2 activation is reversed by simultaneous pharmacological activation of ERK1-2 $(p<0.001$; Fig. $1 a)$.

Figure $1 b$ represents mean percentage total ERK1-2 expression. Mixed-measures ANOVA revealed no significant main effects of group $\left(F_{(5,28)}=1.05, p>0.05\right)$, isoform factor $\left(F_{(1,28)}=\right.$ $0.004, p>0.05)$, or interaction between group and isoform factors $\left(F_{(5,28)}=0.584, p>0.05\right)$, indicating that acute intra-vHipp THC, CBD, or THC+CBD infusion did not alter ERK1-2 expression. The pERK:ERK1-2 expression ratio (transformed from raw pERK and tERK data) is represented in Figure 1c. Mixedmeasures ANOVA revealed a significant main effect of group $\left(F_{(5,28)}=6.88, p<0.001\right)$, but no significant effect of I isoform factor $\left(F_{(1,28)}=0.127, p>0.05\right)$, or interaction between group and isoform factors $\left(F_{(5,28)}=0.334, p>0.05\right)$. Post hoc comparisons using Fisher's LSD revealed that THC significantly increased pERK:ERK1-2 expression ratio relative to VEH ( $p=$ 0.014 ), CBD, and THC $+\mathrm{CBD}$ groups (p's $<0.001$ ). Thus, whereas intra-vHipp THC strongly increases the pERK:ERK1-2 expression ratio relative to $\mathrm{VEH}, \mathrm{CBD}$ shows no effects. In contrast, the THC $+\mathrm{CBD}$ combination significantly downregulates pERK:ERK1-2 expression, an effect that is reversed via coadministration of THC $+\mathrm{CBD}+\mathrm{EPA}(p<0.001)$. Representative Western blots for pERK1-2 and ERK1-2 expression levels in the vHipp are shown in Figure 1d. In Figure 1e, we present a schematic summary of intra-vHipp microinfusion locations for the above described experimental groups.

\section{Intra-vHipp THC and CBD differentially modulate anxiety- like behaviors via local ERK1-2 modulation}

We next examined the potential effects of intra-vHipp THC or CBD on a battery of anxiety-related behavioral assays and the potential role of localized ERK1-2 signaling in these effects. First, using the open field test (see methods), we compared total ambulatory activity after THC/CBD administration and anxietyrelated spatial exploration parameters. Comparing mean total distance traveled after intra-vHipp microinfusion of VEH $(n=$ $11), 10(n=10)$ or $100 \mathrm{ng}$ THC $(n=12), 10(n=9)$ or $100 \mathrm{ng}$ CBD $(n=9)$, or $100 \mathrm{ng} \mathrm{THC}+\mathrm{CBD}(n=8)$ coadministration, one-way ANOVA revealed no significant main effect of group $\left(F_{(5,53)}=0.412, p>0.05\right)$, demonstrating that THC/CBD has no effects on gross motoric behaviors (Fig. 2a).

Next, comparing times spent in the center vs peripheral zones of the test arena during the initial $5 \mathrm{~min}$ of testing revealed differential effects of THC vs CBD on anxiety-like behaviors (Fig. $2 b$ ). First, one-way ANOVA comparing times spent in the center zone between VEH, THC (10 and $100 \mathrm{ng}$ ), CBD (10 and $100 \mathrm{ng}$ ), and THC $+\mathrm{CBD}(100 \mathrm{ng})$ revealed a significant main effect of group $\left(F_{(5,53)}=4.81, p=0.001\right)$. Post hoc comparisons using Fisher's LSD demonstrate that the higher dose of THC (100 ng) decreases time spent in the center zone relative to $\operatorname{VEH}(p=$ 0.046 ), suggesting a dose-dependent anxiogenic effect. In contrast, coadministration of $\mathrm{THC}+\mathrm{CBD}$ induced a significant 

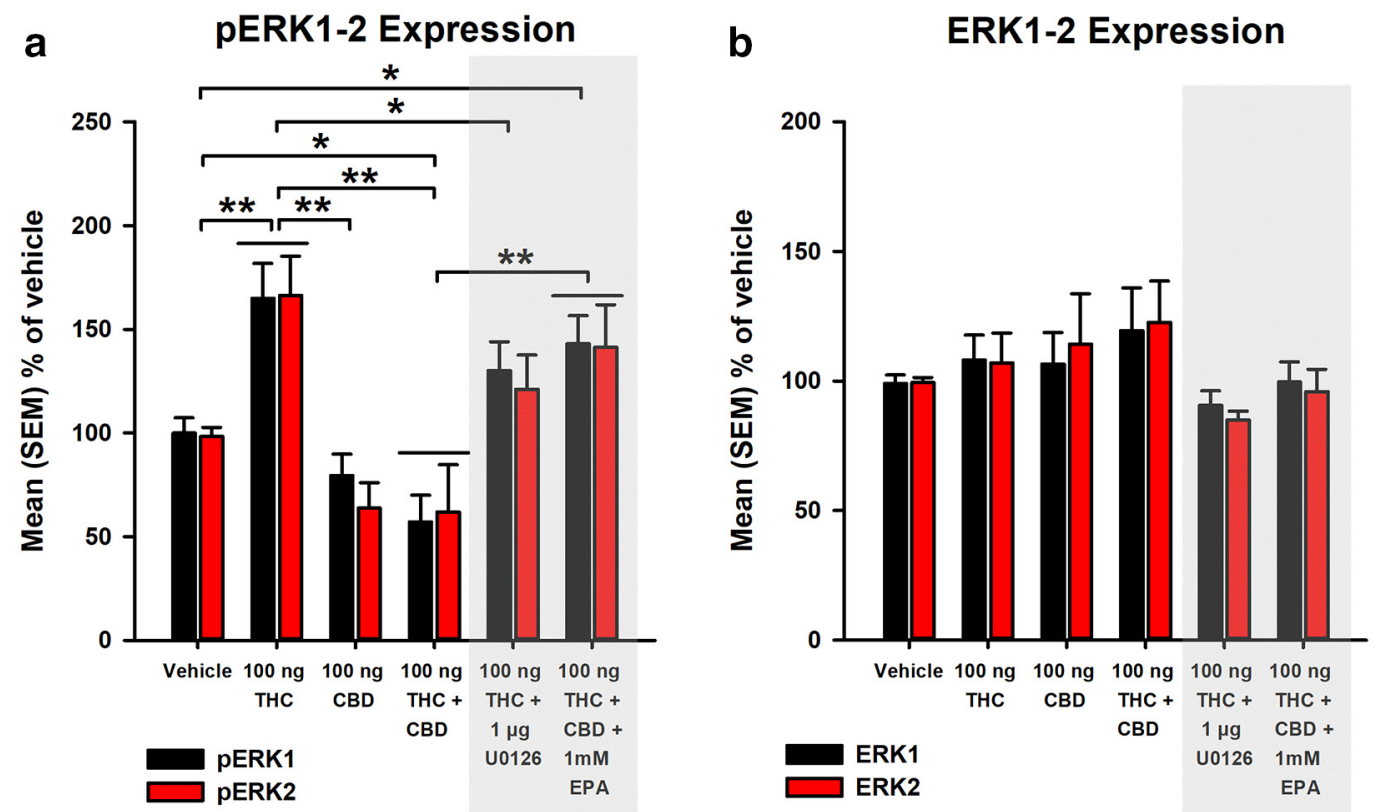

C pERK: ERK1-2 Expression Ratio
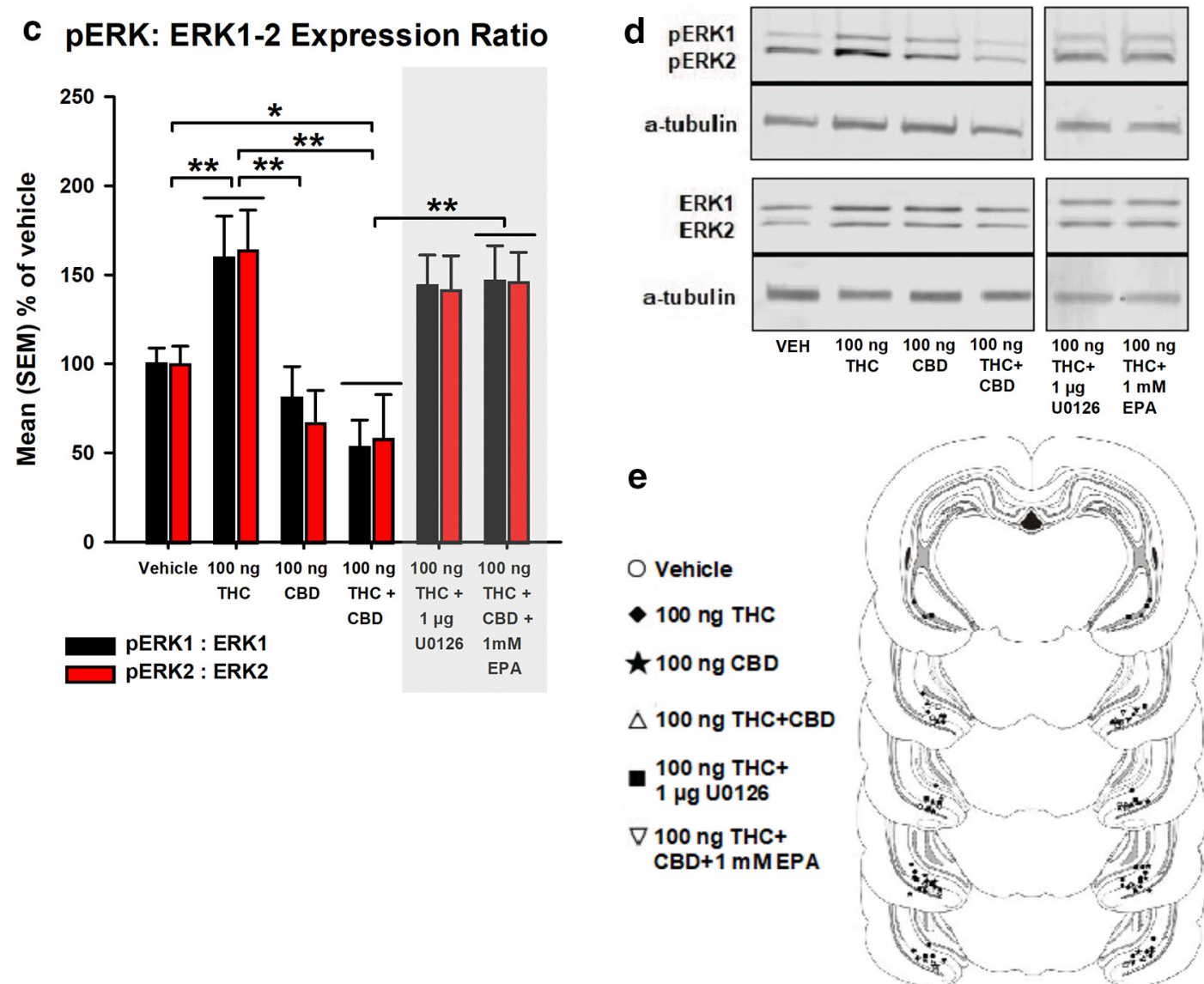

Figure 1. Acute effects of local (a) pERK1-2 expression, (b) ERK1-2 expression, and (c) pERK:ERK1-2 expression ratio in the vHipp of rats treated with VEH, THC (100 ng), CBD (100 ng), THC + CBD $(100 \mathrm{ng}+100 \mathrm{ng})$, THC + U0126 $(100 \mathrm{ng}+1 \mu \mathrm{g})$, and THC + CBD + EPA $(100 \mathrm{ng}+100 \mathrm{ng}+1 \mathrm{~mm})$. (d) Representative Western blot for pERK1-2 and ERK1-2 expression levels, relative to alpha (a)-tubulin in the vHipp. (e) Histological localization of microinfusion sites in the vHipp for each treatment condition (circles: VEH; diamonds: THC; stars: CBD; triangles: THC + CBD; squares: $\mathrm{THC}+$ U0126; and inverted triangles: THC $+(\mathrm{BD}+\mathrm{EPA})$. All drug doses were given in a total volume of $0.5 \mu \mathrm{l} . n=5-6$ rats, Mixed-measures ANOVAs; ${ }^{*} p<0.05 ;{ }^{* *} p<0.01$. Error bars indicate SEM. 

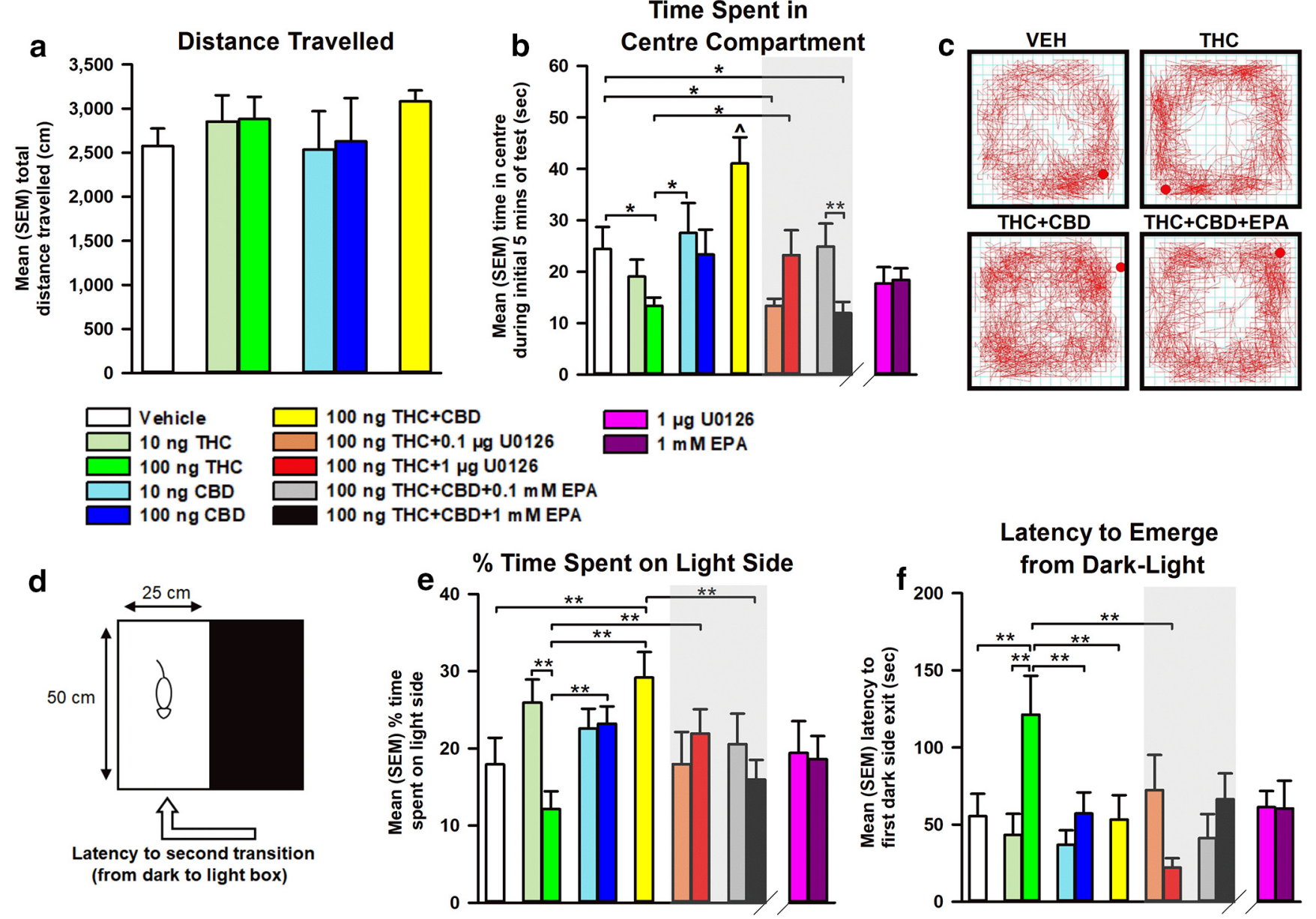

Figure 2. Effects of intra-VHipp VEH, THC (10 and $100 \mathrm{ng})$, CBD (10 and $100 \mathrm{ng}), \mathrm{THC}+\mathrm{CBD}(100 \mathrm{ng}+100 \mathrm{ng})$, THC + U0126 (100 ng + 0.1 and $1 \mu \mathrm{g})$, and THC + CBD + EPA (100 ng + 100 $\mathrm{ng}+0.1$ and $1 \mathrm{~mm}$ ) on (a) distance traveled, and (b) time in center compartment during the open-field test. (c) Representative activity plots for Vehicle, THC (100 ng), THC + CBD (100 ng + $100 \mathrm{ng})$, and THC + CBD + EPA $(100 \mathrm{ng}+100 \mathrm{ng}+1 \mathrm{~mm})$ groups. (d) Depiction of light/dark anxiety test. Effects of intra-vHipp drug treatment on $(\boldsymbol{e})$ percentage time spent in light side, and $(\boldsymbol{f})$ latency to emerge from dark-light in the light/dark anxiety test. $n=8-12$ rats, one-way ANOVAs; ${ }^{*} p<0.05,{ }^{* *} p<0.01, \wedge p<0.05$ relative to all other groups. Error bars indicate SEM.

anxiolytic effect, with rats spending significantly greater time in the center zone relative to $\mathrm{VEH}(p=0.008)$ and THC $(p<0.001)$ groups, indicating that intra-vHipp THC+CBD coadministration engenders opposite effects on anxiety relative to THC.

Given that intra-vHipp THC potently increases pERK1-2 activation (Fig. 1a), we next challenged the anxiogenic effects of intra-vHipp THC with coadministration of the MEK 1-2 inhibitor, U0126. One-way ANOVA comparing VEH $(n=11)$, THC (100 ng; $n=12)$, and $100 \mathrm{ng} \mathrm{THC}+\mathrm{U} 0126(0.1$ and $1 \mu \mathrm{g} ; n=8)$ revealed a significant main effect of group $\left(F_{(3,35)}=3.36, p=\right.$ 0.03 ). Post hoc comparisons using Fisher's LSD revealed that rats receiving THC with a lower dose of U0126 $(0.1 \mu \mathrm{g})$ displayed anxiogenic avoidance of the center zone relative to $\mathrm{VEH}(p=$ $0.028)$. In contrast, rats receiving coadministration of $100 \mathrm{ng}$ THC with a higher dose of U0126 $(1 \mu \mathrm{g})$ spent significantly more time in the center zone relative to THC $(p=0.046)$. Thus, blockade of MEK1-2 signaling dose-dependently blocks the anxiogenic effects of intra-vHipp THC, consistent with its ability to prevent intra-vHipp pERK1-2 activation.

Given our finding that activation of ERK1-2 with EPA reverses the combinatorial effects of THC + CBD on THC-induced pERK1-2 expression levels, we next challenged the anxiolytic effects of THC + CBD on center zone times by coadministration of $\mathrm{THC}+\mathrm{CBD}+\mathrm{EPA}$. One-way ANOVA comparing VEH $(n=11)$, $100 \mathrm{ng} \mathrm{THC}+\mathrm{CBD}(n=8)$, and $100 \mathrm{ng} \mathrm{THC}+\mathrm{CBD}+\mathrm{EPA}(0.1$, and $1 \mathrm{mM} ; n=9, n=8$, respectively) revealed a significant main effect of group $\left(F_{(3,32)}=7.79, p<0.001\right)$. Post hoc comparisons using Fisher's LSD revealed that rats receiving THC + CBD with a higher dose of EPA (1 mM) spent significantly less time in the center zone relative to $\mathrm{VEH}(p=0.032)$, demonstrating that vHipp ERK phosphorylation dose-dependently attenuates the anxiolytic properties of THC+CBD. A separate one-way ANOVA comparing $1 \mu \mathrm{g}$ U0126, and $1 \mathrm{~mm}$ EPA $(n=8)$ alone with the $\mathrm{VEH}$ group revealed no significant main effect of group $\left(F_{(2,25)}=3.058, p>0.05\right)$. Representative activity plots for selected groups are presented in Figure $2 c$.

We next assessed the effects of intra-vHipp THC/CBD using the light/dark box anxiety test (portrayed in Fig. $2 d$ ). Figure $2 e$ represents the mean percentage time spent in the light environment between drug treatment conditions. One-way ANOVA comparing VEH $(n=10), 10(n=9)$ and $100 \mathrm{ng}$ THC $(n=11)$, $10(n=9)$ and $100 \mathrm{ng} \mathrm{CBD}(n=8)$, and $100 \mathrm{ng} \mathrm{THC}+\mathrm{CBD}(n=$ 8 ) groups revealed a significant main effect of group $\left(F_{(5,49)}=\right.$ $4.76, p=0.001$ ). Post hoc comparisons using Fisher's LSD demonstrate dose-dependent effects of THC, as $10 \mathrm{ng}$ THC increases time spent in the light compartment relative to $100 \mathrm{ng} \mathrm{THC}(p<$ $0.001)$, while $100 \mathrm{ng}$ THC reduces this measure relative to $100 \mathrm{ng}$ CBD $(p=0.008)$ and coadministered THC + CBD $(p<0.001)$. Although CBD displays no significant effects, THC + CBD coadministration increases time spent in the light environment com- 
pared with VEH ( $p=0.008$ ), indicating an anxiolytic effect that counteracts the effect of THC.

We next challenged the anxiogenic effects of intra-vHipp THC with coadministration of the MEK 1-2 inhibitor, U0126. One-way ANOVA comparing VEH $(n=10), 100 \mathrm{ng}$ THC $(n=$ $11)$, and $100 \mathrm{ng} \mathrm{THC}+\mathrm{U} 0126(0.1$ and $1 \mu \mathrm{g} ; n=9, n=8$, respectively) revealed no significant main effect of group $\left(F_{(3,34)}=1.68, p>0.05\right)$. However, a planned pre-hoc comparison revealed significantly increased percentage time spent in the light environment by the $100 \mathrm{ng} \mathrm{THC}+1 \mu \mathrm{g}$ U0126 group relative to THC alone $\left(t_{(18)}=-2.57, p=0.01\right)$. Thus, blockade of MEK1-2 signaling dose-dependently mitigates the anxiogenic effects of intra-vHipp THC.

Next, we challenged the behavioral effects of our THC + CBD combination on percentage time spent in the light environment by coadministration of THC $+\mathrm{CBD}+\mathrm{EPA}$. One-way ANOVA comparing VEH $(n=10), 100 \mathrm{ng} \mathrm{THC}+\mathrm{CBD}(n=8)$, and 100 ng THC $+\mathrm{CBD}+\mathrm{EPA}(0.1$ and $1 \mathrm{~mm} ; n=7, n=9$, respectively) revealed a significant main effect of group $\left(F_{(3,30)}=3.05, p=\right.$ 0.044). Post hoc comparisons using Fisher's LSD revealed that whereas rats receiving THC $+\mathrm{CBD}$ with a lower dose of EPA $(0.1$ $\mathrm{mM}$ ) did not differ from $\mathrm{THC}+\mathrm{CBD}$, rats receiving $\mathrm{THC}+\mathrm{CBD}$ with a higher dose of EPA (1 mM) spent significantly less time in the light environment $(p=0.009)$, demonstrating that vHipp pERK1-2 activation dose-dependently attenuates the anxiolytic properties of THC $+\mathrm{CBD}$. A separate one-way ANOVA comparing $1 \mu \mathrm{g} \mathrm{U} 0126$, and $1 \mathrm{~mm}$ EPA $(n=8)$ alone with the VEH group revealed no significant main effect of group $\left(F_{(2,25)}=0.043, p>\right.$ $0.05)$ on percentage time spent in the light environment. Figure $2 f$ represents the mean latency to initial reemergence from the dark to light environment. One-way ANOVA comparing VEH, THC (10 and $100 \mathrm{ng}$ ), CBD (10 and $100 \mathrm{ng}$ ), and THC+CBD (100 ng) revealed a significant main effect of group $\left(\mathrm{F}_{(549)}=3.60, p=\right.$ 0.008). Post hoc comparisons using Fisher's LSD demonstrate that the $100 \mathrm{ng}$ THC group shows increased latency to reemerge into the light environment relative to $\operatorname{VEH}(p=0.006), 100 \mathrm{ng}$ CBD $(p=0.011)$ and THC $+\mathrm{CBD}(p=0.007)$. Thus, intravHipp THC dose-dependently increases anxiogenic avoidance behaviors that are reversed by CBD coadministration.

Comparing groups treated with VEH, $100 \mathrm{ng}$ THC, and 100 ng THC+U0126 $(0.1$ and $1 \mu \mathrm{g})$, one-way ANOVA revealed a significant main effect of group $\left(F_{(3,34)}=4.88, p=0.006\right)$. Post hoc comparisons revealed that rats receiving THC with a higher dose of U0126 $(1 \mu \mathrm{g})$ displayed significantly less latency to reemerge into the light environment relative to THC alone ( $p<$ 0.001 ) and displayed comparable latency to VEH controls. Thus, blockade of intra-vHipp MEK1-2 signaling dosedependently inhibits THC-induced anxiogenic avoidance of a light environment. Furthermore, comparing the $1 \mu \mathrm{g}$ U0126, and $1 \mathrm{mM} \mathrm{EPA}(n=8)$ alone groups with the VEH group using one-way ANOVA revealed no significant main effect of group $\left(F_{(2,25)}=0.046, p>0.05\right)$ on latency to reemerge into the light environment.

\section{Intra-vHipp THC and CBD differentially modulate contextual fear memory formation via local ERK1-2 modulation}

Considering our findings that intra-vHipp THC/CBD opposingly modulate anxiety-related behaviors, we next examined whether THC/CBD could modify the aversive valence of fear memory acquisition using subthreshold foot shock conditioning levels $(0.4 \mathrm{~mA})$. A sample microphotograph of a representative vHipp microinfusion site is shown in Figure $3 a$. First, using a context-dependent fear conditioning assay (see methods), we compared mean percentage time freezing during fear memory testing (Fig. 3b). One-way ANOVA comparing VEH $(n=11), 10$ $(n=8)$ and $100 \mathrm{ng}$ THC $(n=9), 10(n=7)$ and $100 \operatorname{ng} \operatorname{CBD}(n=$ $8)$, and $100 \mathrm{ng}$ THC $+\mathrm{CBD}(n=8)$ groups revealed a significant main effect of group $\left(F_{(5,45)}=3.09, p=0.018\right)$. Post hoc comparisons using Fisher's LSD revealed dose-dependent effects of THC, with the higher dose of THC (100 ng) inducing a significant elevation in percentage time freezing relative to $\operatorname{VEH}(p=0.003)$, $100 \mathrm{ng} \mathrm{CBD}(p=0.038)$ and THC $+\mathrm{CBD}(p=0.001)$. Thus, whereas THC strongly potentiates the aversive salience of contextual cues linked with subthreshold foot shock, THC+CBD coadministration mitigates these effects.

Based upon dose-response curves obtained in our anxietybased assays, we selected coinfusion doses of $1 \mu \mathrm{g}$ U0126, and 1 mM EPA to challenge the effects of THC, and THC + CBD on fear responsivity, respectively. One-way ANOVA comparing VEH $(n=11), 100 \mathrm{ng}$ THC $(n=9)$, and $100 \mathrm{ng}$ THC $+\mathrm{U} 0126(n=9)$ groups revealed a significant main effect of group $\left(F_{(2,26)}=4.18\right.$, $p=0.027)$. Post hoc comparisons using Fisher's LSD revealed that coadministered $100 \mathrm{ng}$ THC $+\mathrm{U} 0126$ reduced percentage time spent freezing relative to $100 \mathrm{ng} \mathrm{THC}(p=0.034)$, demonstrating that MEK1-2 blockade counteracts THC-induced potentiation of fear responsivity.

We next challenged the antagonistic effect of intra-vHipp $\mathrm{THC}+\mathrm{CBD}$ coadministration by coinfusing THC $+\mathrm{CBD}+\mathrm{EPA}$. One-way ANOVA comparing VEH $(n=11), 100 \mathrm{ng}$ THC + CBD $(n=8)$, and $100 \mathrm{ng} \mathrm{THC}+\mathrm{CBD}+1 \mathrm{~mm} \mathrm{EPA}(n=10)$ revealed no significant main effect of group $\left(F_{(2,26)}=2.91, p>0.05\right)$. However, a planned pre-hoc comparison revealed that $\mathrm{THC}+\mathrm{CBD}+$ EPA coadministration significantly increased percentage time spent freezing relative to THC $+\mathrm{CBD}\left(t_{(16)}=-2.19, p=0.043\right)$, demonstrating that vHipp pERK1-2 upregulation mitigates relief of fear-responsivity by THC $+\mathrm{CBD}$. In addition, a separate oneway ANOVA comparing $1 \mu \mathrm{g}$ U0126, and $1 \mathrm{~mm}$ EPA alone $(n=$ 8) relative to VEH revealed no significant main effect of group $\left(F_{(2,24)}=2.71, p>0.05\right)$.

\section{THC and CBD differentially regulate context-independent fear memory processing via interactions with ERK1-2 signaling in the vHipp}

Considering that the vHipp has previously been implicated in context-independent associative memory formation (Kramar et al., 2017), we next tested the effects of intra-vHipp THC/CBD administration and the impact of pERK1-2 signaling on olfactory-cue fear memory formation using a subthreshold fear conditioning protocol (see methods). Based upon dose-response curves obtained in our context-dependent fear conditioning task (Fig. 3b), we selected doses of $100 \mathrm{ng}$ THC, $100 \mathrm{ng}$ CBD, and 100 ng THC + CBD for intra-vHipp microinfusions. The mean percentage time freezing to $\mathrm{CS}^{-}$and $\mathrm{CS}^{+}$presentations during testing are represented in Figure $3 c$. Mixed-measures ANOVA comparing VEH, THC, CBD, and THC + CBD groups $(n=8)$ revealed a significant main effect of group $\left(F_{(3,28)}=13.36, p<\right.$ 0.001 ), and a significant main effect of conditioned stimulus factor $\left(F_{(1,28)}=24.67, p<0.001\right)$, but not an interaction between group and conditioned stimulus factors. Post hoc comparisons using Fisher's LSD revealed that THC significantly increases percentage time freezing to $\mathrm{CS}^{-}$and $\mathrm{CS}^{+}$relative to VEH $(p=$ $0.022 ; p<0.001), \mathrm{CBD}(p=0.007 ; p<0.001)$, and THC $+\mathrm{CBD}$ $(p=0.007 ; p<0.001)$ groups. Thus, whereas intra-vHipp THC indiscriminately potentiates fear responsivity to associative fear- 

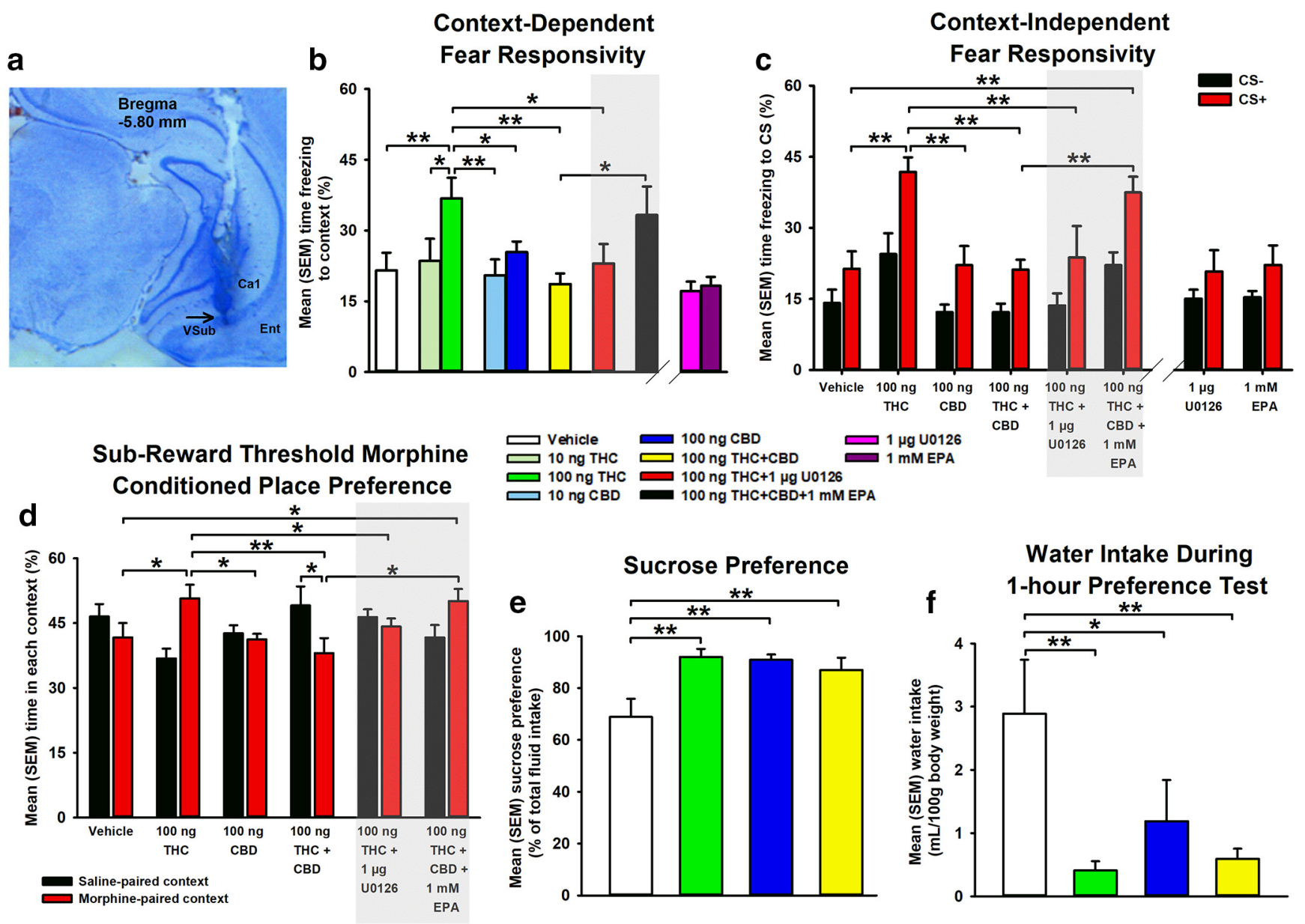

Water Intake During

Sucrose Preference
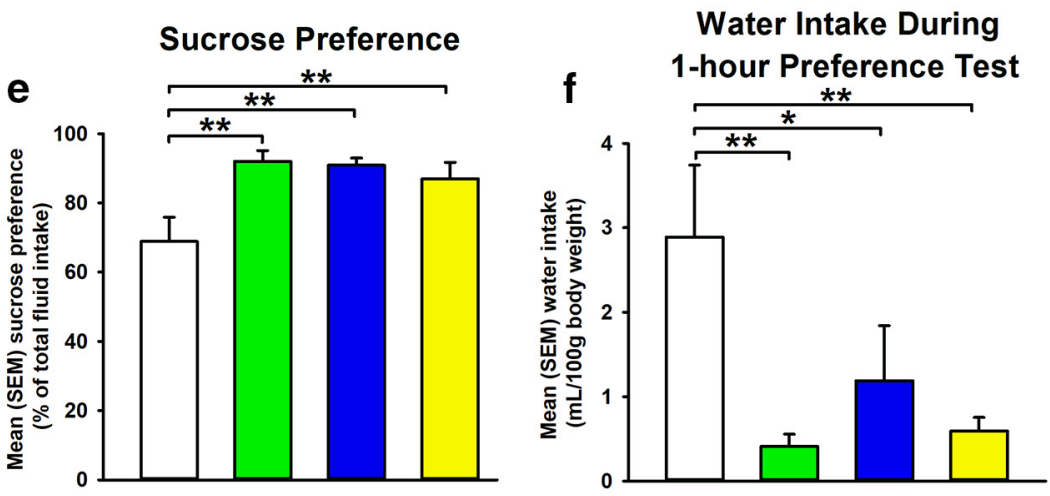

Figure 3. Effects of intra-vHipp drug treatments on rewarding and aversive memory formation. $\boldsymbol{a}$, Photomicrograph demonstrating a representative vHipp microinfusion site. $\boldsymbol{b}$, Effects of intra-VHipp VEH, THC (10 and $100 \mathrm{ng})$, CBD (10 and $100 \mathrm{ng})$, THC + CBD (100 ng + $100 \mathrm{ng})$, THC + U0126 (100 ng + $1 \mu \mathrm{g})$, and THC + CBD + EPA (100 ng + $100 \mathrm{ng}+1 \mathrm{~mm})$ on percentage of time freezing to contextual, and (c) olfactory-associative $\mathrm{CS}^{+}$and $\mathrm{CS}^{-}{ }^{-}$cues, $(\boldsymbol{d})$ percentage of time spent in morphine and saline-paired contexts during subreward threshold morphine $(0.05 \mathrm{mg} / \mathrm{kg}$, i.p.) CPP testing, $(\boldsymbol{e})$ sucrose preference, and $(\boldsymbol{f})$ water intake during sucrose preference testing. Intra-vHipp drug infusion occurred before each conditioning session. $n=7-11$ rats, one-way, or mixed-measures ANOVAs; ${ }^{*} p<0.05,{ }^{* *} p<0.01$. Error bars indicate SEM.

conditioned cues, THC + CBD coadministration mitigates these conditioned effects.

We next examined the impact of pERK1-2 signaling on THC-induced changes in context-independent fear responsivity. Mixed-measures ANOVA comparing VEH, THC, and THC+ U0126 groups $(n=8)$ revealed significant main effects of group $\left(F_{(2,21)}=8.11, p=0.002\right)$, and conditioned stimulus factor $\left(F_{(1,21)}=13.18, p=0.002\right)$, but not an interaction between group and conditioned stimulus factors. Post hoc comparisons using Fisher's LSD revealed that rats treated with THC+U0126 display less percentage time freezing to the $\mathrm{CS}^{+}$relative to THC alone $(p=0.004)$, and do not differ in $\mathrm{CS}^{-}$freezing percentage relative to VEH. Thus, inhibition of pERK1-2 signaling is sufficient to counteract THC-induced potentiation of fear responsivity.

Next, we examined the potential role of pERK1-2 signaling in the inhibitory action of $\mathrm{CBD}$ coadministration on THC-induced fear responsivity by coadministering THC $+\mathrm{CBD}+\mathrm{EPA}$. Mixedmeasures ANOVA comparing $\mathrm{VEH}, \mathrm{THC}+\mathrm{CBD}$, and THC+ CBD+EPA groups $(n=8)$ revealed significant main effects of group $\left(F_{(2,21)}=10.61, p<0.001\right)$, and conditioned stimulus factor $\left(F_{(1,21)}=28.54, p<0.001\right)$, but no interaction between group and conditioned stimulus factors. Post hoc comparisons indicated coinfusion of THC $+\mathrm{CBD}+\mathrm{EPA}$ increased percentage time freezing to $\mathrm{CS}^{-}$and $\mathrm{CS}^{+}$relative to $\mathrm{THC}+\mathrm{CBD}(p=0.026$; $p<0.001$ ), indicating that CBD coadministration rescues THCinduced associative fear memory formation via inhibition of vHipp pERK1-2 activation. Additionally, a separate mixedmeasures ANOVA comparing $1 \mu \mathrm{g}$ U0126, and $1 \mathrm{~mm}$ EPA alone $(n=8)$ relative to VEH revealed a significant main effect of conditioned stimulus factor $\left(F_{(1,21)}=10.25, p=0.004\right)$, but no significant main effect of group or interaction between group and conditioned stimulus factors.

Intra-vHipp THC and CBD produce opposite effects on opioid reward processing via local ERK1-2 modulation In addition to modulation of fear-related associative memory, using a morphine-dependent CPP procedure, we recently reported that vHipp CB1R activation can strongly potentiate opioid reward memory salience (Loureiro et al., 2015). Therefore, we used a subreward threshold conditioning doses of morphine $(0.05 \mathrm{mg} / \mathrm{kg}$, i.p.) with an unbiased CPP design (see methods) assessing mean percentage time spent in the morphine-paired and saline-paired contexts during CPP testing (Fig. 3d). Mixedmeasures ANOVA comparing VEH, THC, CBD, and THC + CBD groups $(n=7)$ revealed a significant interaction between group and context factors $\left(F_{(3,24)}=3.38, p=0.035\right)$, but no significant main effect of group, or context factor. Post hoc comparisons using Fisher's LSD indicated that THC significantly 
increased percentage time spent in the morphine context relative to $\mathrm{VEH}(p=0.035), \mathrm{CBD}(p=0.028)$, and THC $+\mathrm{CBD}(p=$ 0.007 ) groups. Furthermore, a planned pre-hoc comparison revealed that rats receiving $\mathrm{THC}+\mathrm{CBD}$ coadministration demonstrated greater percentage time spent in the saline vs morphine-paired contexts $\left(t_{(12)}=-1.93, p=0.039\right)$. Thus, whereas intra-vHipp THC potentiates the reward salience of morphine, THC $+\mathrm{CBD}$ coadministration reverses this effect, producing aversion to morphine-paired contexts. We also separately analyzed both the average time spent in the center gray compartment (one-way ANOVA: $F_{(3,24)}=1.39, p>0.05$ ), as well as the average time spent in either conditioning environment (mixed-measures ANOVA: $F_{(3,48)}=0.68, p>0.05$ ) to examine possible contextual bias, but the analyses revealed no significant differences between groups.

We next examined whether the ability of intra-vHipp THC to modulate morphine-dependent reward processing may depend upon local pERK1-2 signaling. Mixed-measures ANOVA comparing $\mathrm{VEH}, \mathrm{THC}$, and $\mathrm{THC}+\mathrm{U} 0126(n=8)$ groups revealed a significant interaction between group and Context $\left(F_{(2,19)}=\right.$ $4.02, p=0.035)$. Post hoc comparisons using Fisher's LSD showed that rats receiving $\mathrm{THC}+\mathrm{U} 0126$ did not differ from $\mathrm{VEH}$ in percentage time spent in the morphine context, suggesting that THC potentiates the rewarding properties of morphine via local pERK1-2 signaling. We also assessed the role of pERK1-2 signaling on the opposing actions of $\mathrm{THC}+\mathrm{CBD}$ on $\mathrm{CPP}$ via coadministration of THC $+\mathrm{CBD}+\mathrm{EPA}$. Mixed-measures ANOVA comparing $\mathrm{VEH}, \mathrm{THC}+\mathrm{CBD}$, and $\mathrm{THC}+\mathrm{CBD}+\mathrm{EPA}$ groups $(n=7)$ revealed a significant main effect of group $\left(F_{(2,18)}=4.24\right.$, $p=0.031)$. Post hoc comparisons indicated that relative to rats receiving $\mathrm{VEH}(p=0.022)$, those receiving $\mathrm{THC}+\mathrm{CBD}+\mathrm{EPA}$ increased percentage time spent in the morphine context. Therefore, $\mathrm{CBD}$ coadministration reverses the potentiation of reward memory salience induced by intra-vHipp THC via local pERK1-2 inhibition.

\section{THC, CBD, and their combination enhance preference for a low-concentration sucrose solution}

We next examined the effects of THC, CBD, and their combination on responses toward a natural reward using the sucrose preference assay (see methods). Mean preference for 2\% sucrose solution is represented in Figure $3 e$. One-way ANOVA comparing VEH $(n=7)$, THC $(n=8), \mathrm{CBD}(n=7)$, and THC+CBD $(n=7 ; 100 \mathrm{ng}$ each) revealed a significant main effect of group $\left(F_{(3,25)}=5.61, p=0.004\right)$, and post hoc comparisons using Fisher's LSD revealed that THC ( $p=0.001), \operatorname{CBD}(p=0.002)$, and THC + CBD $(p=0.01)$ each significantly increase sucrose preference compared with VEH. Similar results were observed when comparing water intake between groups during the sucrose preference assay (main effect of group $\left(F_{(3,25)}=4.51, p=0.012\right)$; Fig. $3 f)$. Total caloric intake during testing did not differ between groups $\left(\left(F_{(3,25)}=2.48, p=0.084\right)\right)$. Thus, intra-vHipp THC, $\mathrm{CBD}$, and combined $\mathrm{THC}+\mathrm{CBD}$ each selectively enhance preference for a low-concentration sucrose reward by decreasing water intake, without altering total caloric consumption or food intake.

\section{Intra-vHipp THC elicits a hyperactive VTA DA activity state via ERK1-2 activation and is blocked by CBD}

We next performed intra-VTA extracellular single-unit recordings to determine whether VTA DA neuronal activity states are modulated by intra-vHipp VEH (cells/animals; $n=13 / 5$ ), THC $(n=19 / 6), \operatorname{CBD}(n=12 / 4)$, combined THC $+\mathrm{CBD}(n=16 / 6)$, or local pERK1-2 activity using THC+U0126 ( $n=14 / 5)$. Sample microphotographs of a representative vHipp microinfusion location and VTA neuronal recording site are shown in Figure 4, $a$ and $b$. Neuronal activity profiles after intra-vHipp microinfusion treatments are summarized in Figure $4 c$. Figure $4 d$ represents mean frequency change (expressed as percentage relative to baseline) in VTA DA neurons, and one-way ANOVA revealed a significant main effect of group $\left(F_{(4,69)}=3.54 p=0.011\right)$. Post hoc comparisons using Newman-Keuls' test revealed that VTA DA neuronal firing frequencies were significantly increased after intra-vHipp THC relative to VEH $(p=0.028), \mathrm{CBD}(p<0.001)$, $\mathrm{THC}+\mathrm{CBD}(p=0.011)$ groups. One-way ANOVA comparing mean VTA DA bursting activity change (expressed as bursts/min, percentage relative to baseline) between VEH (cells/animals; $n=$ $11 / 5), \operatorname{THC}(n=14 / 6), \operatorname{CBD}(n=10 / 4), \operatorname{THC}+\operatorname{CBD}(n=16 / 6)$, and THC+U0126 $(n=11 / 5)$ revealed similar results (significant main effect of group $\left(F_{(4,57)}=2.61, p=0.045\right)$; Fig. $\left.4 e\right)$. Thus, whereas THC increases VTA DA neuronal frequency and phasic bursting rates through a vHipp pERK1-2 dependent substrate, $\mathrm{THC}+\mathrm{CBD}$ coadministration mitigates these changes. Rastergrams demonstrating effects on firing frequency according to treatment with $\mathrm{THC}$ alone, or combined $\mathrm{THC}+\mathrm{CBD}$ are represented in Figure $4, f$ and $g$, respectively.

\section{Intra-vHipp THC and CBD reduce VTA non-DA neuronal activity}

Putative non-DA neurons isolated in the VTA were also analyzed. Neuronal activity profiles after intra-vHipp microinfusion treatments are summarized in Figure $5 a$. Figure $5 b$ represents mean frequency change (expressed as percentage relative to baseline) in VTA non-DA neurons. One-way ANOVA comparing VEH (cells/animals, $n=15 / 5)$, THC $(n=14 / 6)$, CBD $(n=11 / 4)$, $\mathrm{THC}+\mathrm{CBD}(n=11 / 6)$, and THC+U0126 groups $(n=14 / 5)$ revealed a significant main effect of group $\left(F_{(4,60)}=3.01, p=\right.$ 0.025). Post hoc comparisons using Newman-Keuls' test reveal that THC $(p=0.008)$ and CBD $(p=0.041)$ each reduce VTA non-DA neuronal frequency rates relative to $\mathrm{VEH}$. Coadministration of THC $+\mathrm{CBD}(p=0.007)$, and THC+U0126 $(p=$ 0.019 ) each mitigated the actions of THC. Rastergrams demonstrating effects on firing frequency according to treatment with THC alone, or combined THC $+\mathrm{CBD}$ are shown in Figure 5, c and $d$, respectively.

\section{Intra-vHipp THC and CBD exert distinct control over VTA oscillatory frequencies}

LFP recordings in the VTA were obtained simultaneously with single-unit activity. The strength of the oscillations was assessed by calculating power spectral densities (PSDs; window length $=$ $2 \mathrm{~s}$; shift $=0.5 \mathrm{~s}$ ) during the 5 min preinfusion and postinfusion recording epochs, averaging PSDs within epochs and subsequently normalizing the averaged PSD to the total power (normalized total power $=1$; frequencies values between 59 and 61 $\mathrm{Hz}$ were excluded to avoid contamination with $60 \mathrm{~Hz}$ noise from power line). Figure $6 a$ depicts representative VTA spectrograms in the preinfusion and postinfusion recording epochs after intravHipp microinfusions of $100 \mathrm{ng}$ THC, and Figure $6 b$ represents the average normalized power spectra corresponding to VTA LFP of rats receiving VEH or THC. Difference scores calculated between preinfusion and postinfusion epochs for $\beta, \gamma$, and $\varepsilon$ power spectra oscillations, calculated as the sum of power values for frequencies between 15 and $30 \mathrm{~Hz}, 30$ and $80 \mathrm{~Hz}$, and 90 and 100 $\mathrm{Hz}$ respectively, after infusion of $\mathrm{VEH}(n=16)$, THC $(n=15)$, 


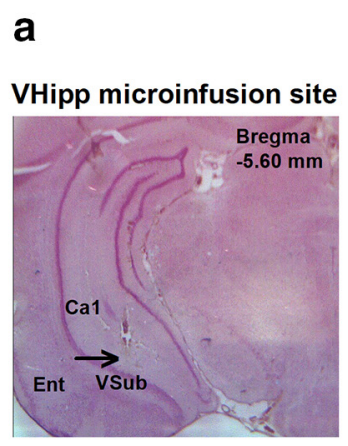

b

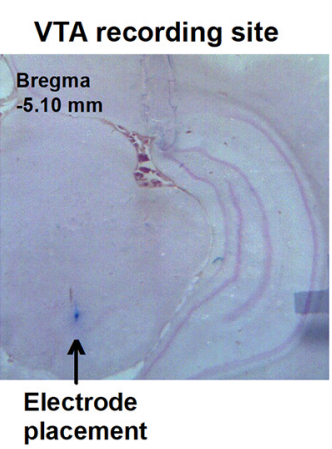

placement

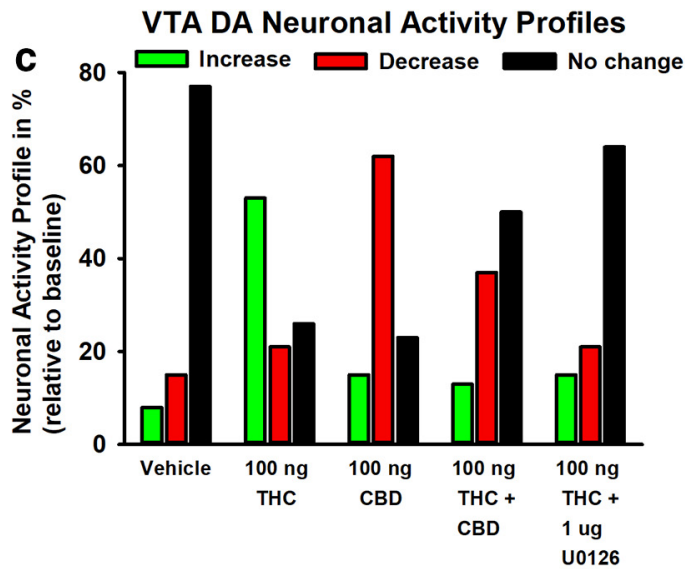

e
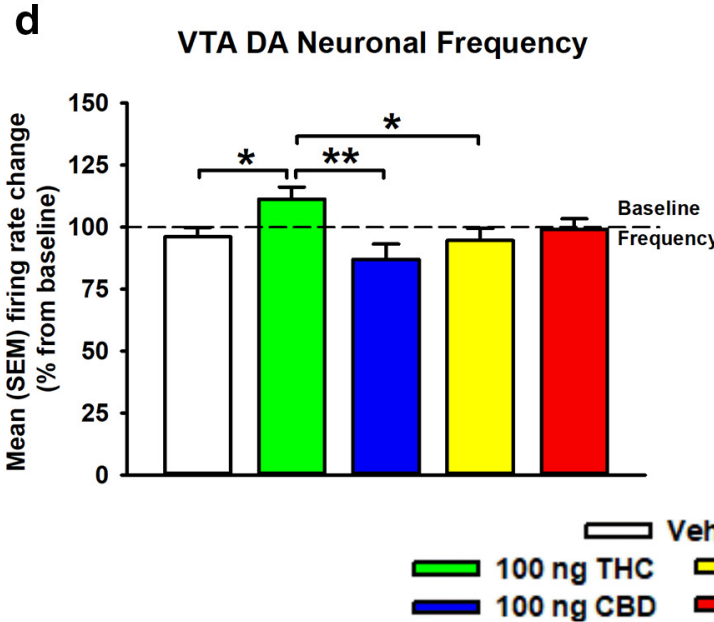

Vehicle

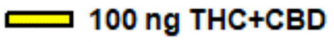

$100 \mathrm{ng}$ THC+1 U0126

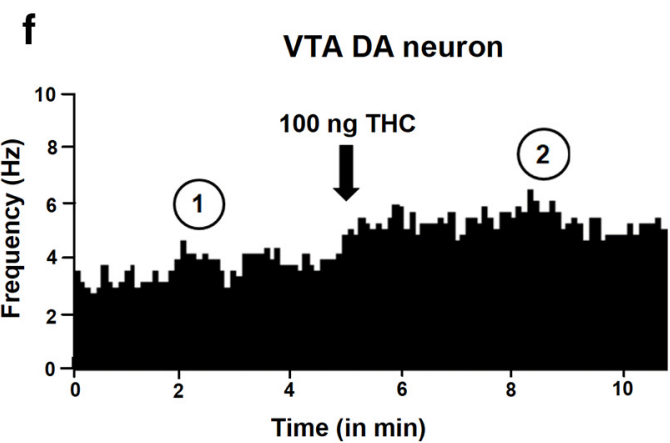

g
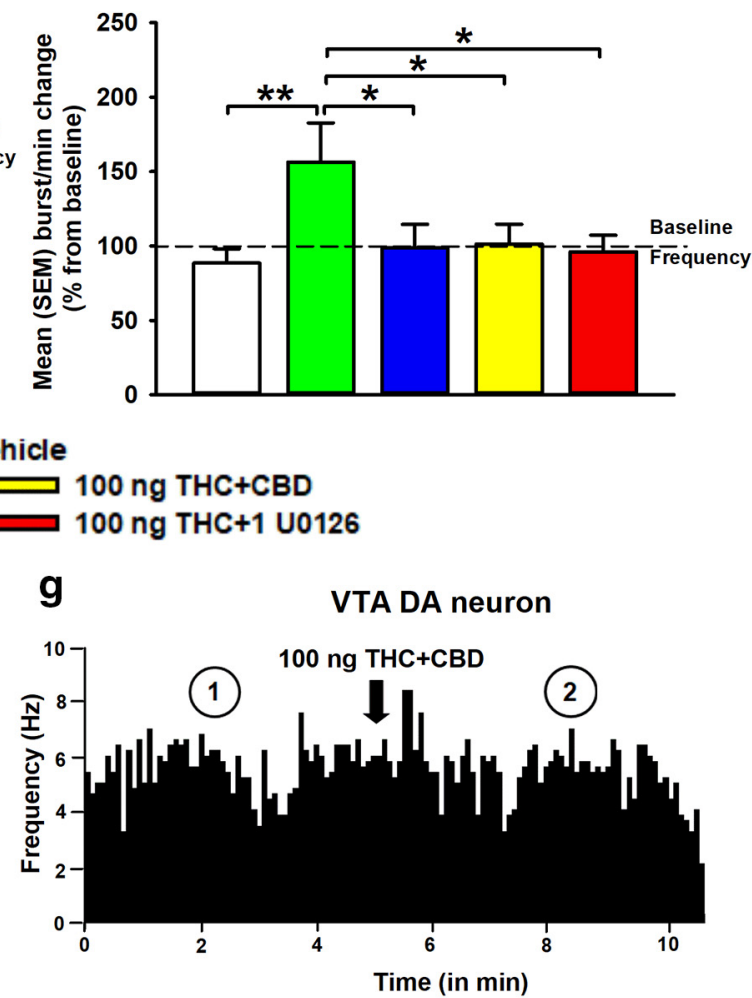

(1)
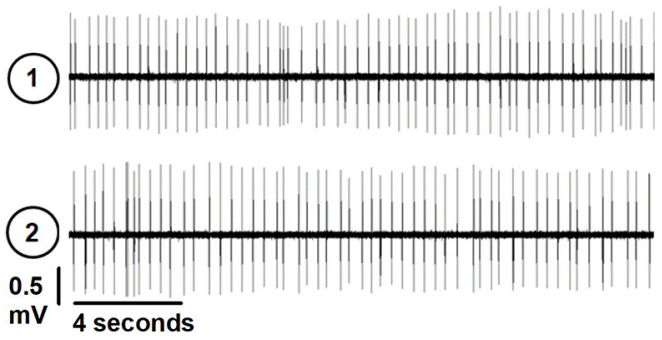

Figure 4. Effects of intra-VHipp VEH, THC (100 ng), CBD (100 ng), THC + CBD (100 ng + 100 ng), and THC + U0126 (100 ng + $1 \mu \mathrm{g})$ exposure on spontaneous VTA putative DA neuronal activity. $\boldsymbol{a}$, Microphotograph of a representative VTA microinfusion site. $\boldsymbol{b}$, VTA neuronal recording placement. $\boldsymbol{c}$, Summary of the VTA DA neuronal activity profile (i.e., number of cells that increased, decreased, or did not change their firing frequency after microinfusions). $\boldsymbol{d}, \boldsymbol{e}$, Consequences of intra-vHipp drug treatments on VTA DA neuronal firing frequency $(\boldsymbol{d})$ and burst rate $(\boldsymbol{e}) . \boldsymbol{f}, \boldsymbol{g}$, Representative histogram showing the increase in response activity of one DA neuron upon microinfusion of THC $(\boldsymbol{f})$ and example of a DA neuron showing no change in activity level after THC + CBD coinfusion $(\boldsymbol{g})$. For each panel, inset shows (1) the action potential waveform of the selected neuron and (2) the activity patterns recorded before (baseline activity) and after the microinfusions respectively. $n=12-16$ cells from $n=4-6$ rats, one-way ANOVAs; ${ }^{*} p<0.05,{ }^{* *} p<0.01$. Error bars indicate SEM. 

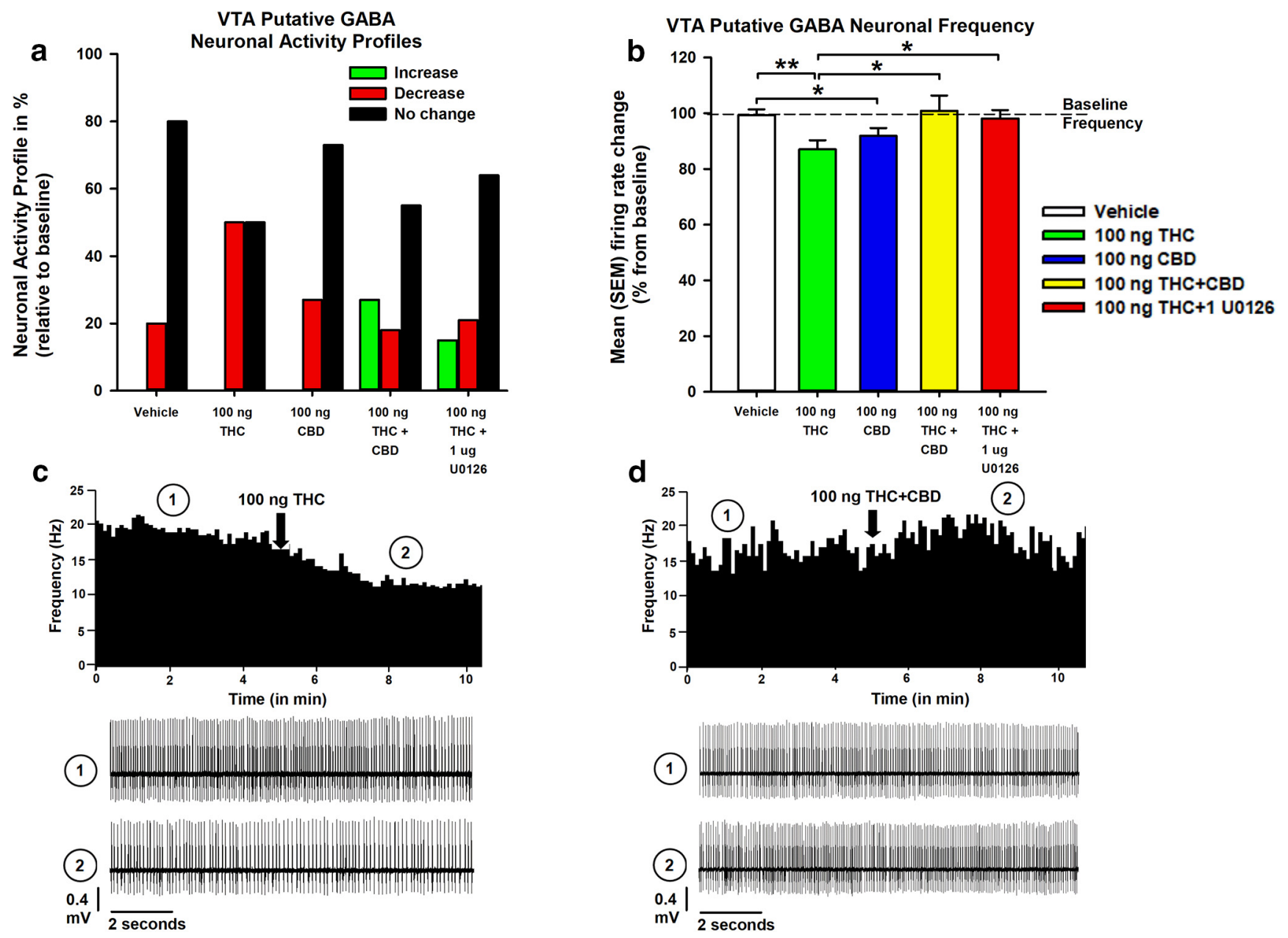

Figure 5. Effects of intra-vHipp VEH, THC (100 ng), CBD (100 ng), THC + CBD (100 ng $+100 \mathrm{ng})$, and THC + U0126 (100 ng $+1 \mu \mathrm{g})$ exposure on VTA non-DA (putative GABA interneurons) activity. $\boldsymbol{a}$, Summary of the VTA non-DA neuronal activity profile (i.e., number of cells that increased, decreased, or did not change their firing frequency after microinfusions). $\boldsymbol{b}$, Consequences of intra-vHipp drug infusion on VTA GABA neuronal firing frequency. $\boldsymbol{c}, \boldsymbol{d}$, Representative histogram showing the decrease in tonic firing frequency of a single VTA non-DA neuron upon microinfusion of THC (c) and example of a non-DA neuron showing a nonsignificant increase in frequency levels after THC + CBD coinfusion (d). For each panel, inset shows (1) the action potential waveform of the selected neuron and (2) the activity patterns recorded before (baseline activity) and after the microinfusions, respectively. $n=11-15$ cells from $n=4-6$ rats, one-way ANOVAs; ${ }^{*} p<0.05$, ${ }^{* *} p<0.01$. Error bars indicate SEM.

$\mathrm{CBD}(n=16), \mathrm{THC}+\mathrm{CBD}(n=15)$, and THC $+\mathrm{U} 0126(n=12)$ are represented in Figure 6, $c$ to $e$.

One-way ANOVA examining oscillatory power changes in the $\beta$ frequency range upon intra-vHipp THC revealed a significant main effect of group $\left(F_{(4,69)}=6.70, p<0.001\right)$, and post hoc comparisons using Newman-Keuls' test show that THC significantly increases $\beta$ power relative to all other groups $(p<0.001$ for all). Comparable results are observed within $\gamma$, and $\varepsilon$ frequency ranges ( $\gamma$ : main effect of group $\left(F_{(4,69)}=5.22, p<0.001\right)$; $\varepsilon$ : main effect of group $\left.\left(F_{(4,69)}=2.99, p=0.024\right)\right)$. These data suggest that local pERK1-2 activation regulates THC-induced dysregulation of intra-VTA $\beta, \gamma$, and $\varepsilon$ frequencies, consistent with the observed VTA neuronal frequency abnormalities induced by THC.

\section{Discussion}

Emerging evidence suggests that CBD possesses antipsychotic properties and can mitigate many of the psychotropic side effects of THC (Bhattacharyya et al., 2010; Renard et al., 2016). Cannabis strains and extracts containing high-THC and low-CBD concentrations are linked to increased neuropsychiatric risk (Di Forti et al., 2009; Schubart et al., 2011; Englund et al., 2013; Beale et al.,
2018), underscoring the importance of CBD as a mitigating factor in reducing THC-related neuropsychiatric side effects. However, little is understood regarding how CBD produces these mitigating effects at the neuronal, molecular and behavioral levels. We report that intra-vHipp THC increases VTA DA frequency and bursting rates while concomitantly decreasing GABAergic neuronal activity, and increasing the magnitude of intra-VTA $\beta, \gamma$, and $\varepsilon$ oscillatory frequencies via a vHipp pERK1-2 mediated mechanism. Whereas THC induces an anxiogenic phenotype and distorts reward and aversion-related salience attribution, we found that $\mathrm{THC}+\mathrm{CBD}$ coadministration blocked these effects through differential modulation of local vHipp pERK1-2 signaling states.

Consistent with evidence linking hippocampal CB1R stimulation with pERK1-2 activation (Derkinderen et al., 2003), we observed strongly upregulated local pERK1-2 expression after intra-vHipp THC administration. This effect was selectively blocked by coadministration of the MEK1-2 inhibitor, U0126. Although CBD alone produced no effect on pERK1-2 expression, coadministered THC $+\mathrm{CBD}$ reversed the effects of THC and significantly downregulating $\mathrm{pERK} 1-2$ expression relative 


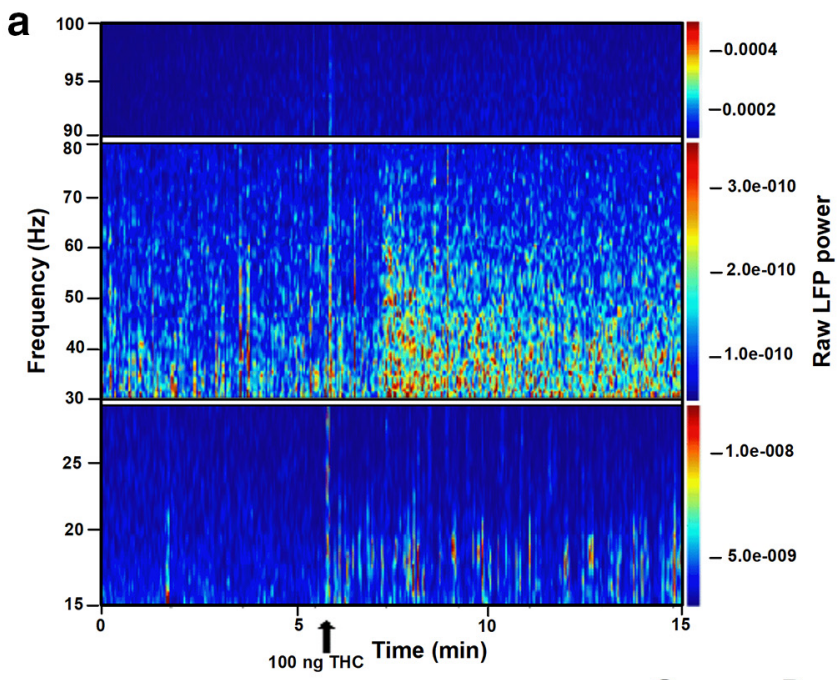

Beta Power Change
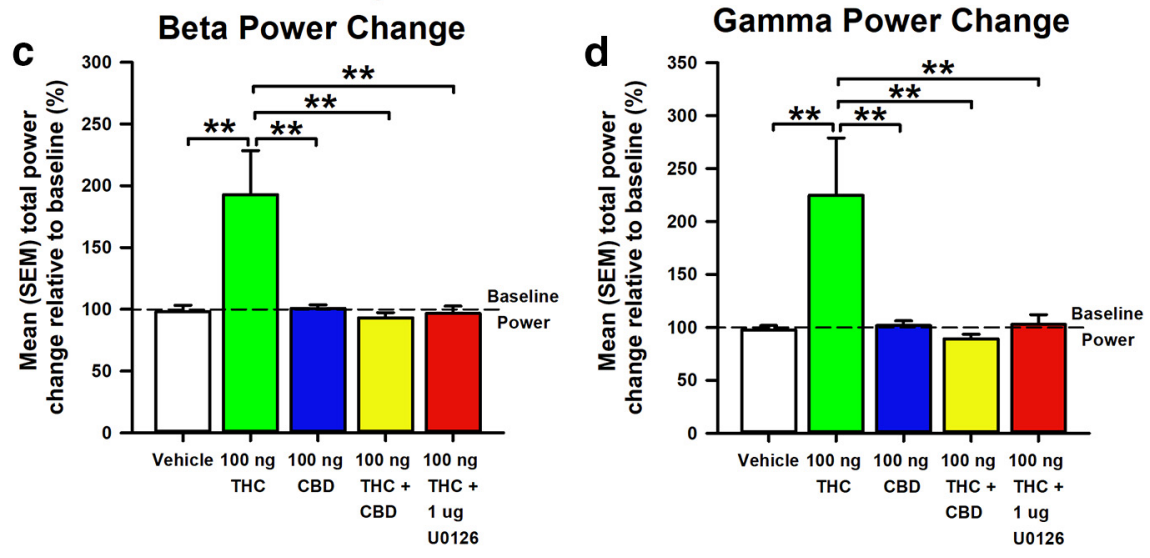

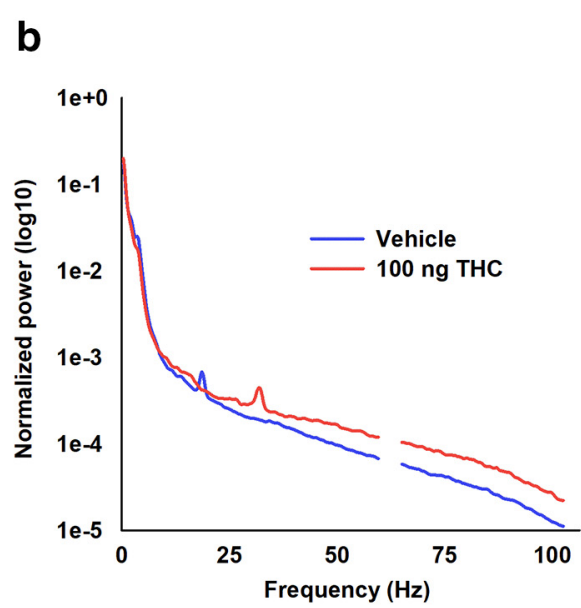

Epsilon Power Change

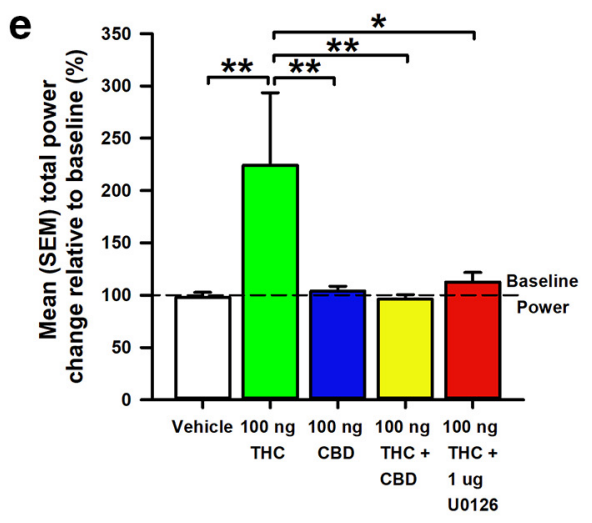

Figure 6. Effects of intra-vHipp VEH, THC (100 ng), CBD (100 ng), THC + CBD (100 ng + $100 \mathrm{ng})$, and THC + U0126 (100 ng + $1 \mu \mathrm{g})$ exposure on $\beta(15-30 \mathrm{~Hz}), \gamma(30-80 \mathrm{~Hz})$, and $\varepsilon(90-100 \mathrm{~Hz})$ oscillatory power in the VTA of a urethane anesthetized rat. $\boldsymbol{a}$, Representative spectrogram showing temporal changes in the power of intra-VTA oscillations at different frequencies. The power values are color-coded as indicated on the right-hand side insets. A peak at $\sim 60 \mathrm{~Hz}$ reflect power line frequency and the LFP power values for frequencies between 59 and $61 \mathrm{~Hz}$ were excluded from further analysis. $\boldsymbol{b}$, Average normalized power spectra corresponding to LFP of VEH-treated (blue) and THC-treated (red) rats. Note the increased power of $\beta$ ( $15-30 \mathrm{~Hz}$ ), $\gamma(30-80 \mathrm{~Hz}$ ), and $\varepsilon$ $(90-100 \mathrm{~Hz})$ bands in THC-treated rats. $c-e$, Bar graphs summarizing the average total power change of the different frequency bands after intra-vHipp drug microinfusion. $n=12-16$ from $n=$ $4-6$ rats, one-way ANOVAs; ${ }^{*} p<0.05,{ }^{* *} p<0.01$. Error bars indicate SEM.

to THC and VEH groups. This is the first evidence, to our knowledge, that combined THC $+\mathrm{CBD}$ functionally reverses the actions of THC on local pERK1-2 signaling. Given that CBD modulates ERK signaling through a very narrow dose range (Solinas et al., 2013), primarily via cannabinoid and vanilloid receptor-independent mechanisms (McPartland et al., 2007), indirect mechanisms such modulation of endocannabinoid signaling could underlie the effects by CBD on pERK1-2 activity in the current study. In addition, CBD's inhibitory effect on THC-induced pERK1-2 activation was blocked by local application of the ERK activator, EPA, further demonstrating the importance of vHipp ERK phosphorylation in the functional effects of THC and CBD. Interestingly, at the molecular level, the results of the current study resemble postmortem assays conducted with brains of schizophrenia cohorts (Kozlovsky et al., 2004).

The ERK signal transduction cascade is a critical regulator of synaptic plasticity, DA and glutamate neurotransmission, and affective processing, each of which are dysregulated in schizophrenia and related disorders (Yuan et al., 2010). ERK expression is functionally coupled with hippocampal CB1R stimulation, which can overdrive mesolimbic DA transmission and disrupt gating of emotionally salient information (Derkinderen et al.,
2003; Loureiro et al., 2015). Given that vHipp CB1Rs are highly expressed on cholecystokinin (CCK)-positive GABAergic interneurons (Derkinderen et al., 2003; Takács et al., 2015), direct agonism of these CB1Rs represents one potential mechanism driving THC-induced pERK1-2 activation.

Alterations in hippocampal pERK1-2 signaling are associated with exposure to stressors and anxiety behaviors, and ERK phosphorylation is importantly involved in fear-related learning and memory processing (Selcher et al., 2003; Huh et al., 2009; Ritov et al., 2014). Thus, our findings that vHipp THC induces a dramatic increase in local ERK1-2 phosphorylation states suggests a potential molecular mechanism by which THC might serve to amplify the salience of incoming affective contextual stimuli. Indeed, distortions of fear-related associative memory are observed after THC administration in the nucleus accumbens (Fitoussi et al., 2018) and also upon CB1R stimulation in the vHipp or basolateral nucleus of the amygdala (Laviolette and Grace, 2006; Loureiro et al., 2015). Activation of vHipp CB1Rs reduce local GABA release, thereby increasing hippocampal excitatory output (Hájos and Freund, 2002; Ivanov et al., 2006), and the psychotomimetic effects of THC in both humans and rodents have been shown to be directly related to striatal glutamate influx (Loureiro et al., 2015; Colizzi et al., 2019). 
Behaviorally, we observed dose-dependent, biphasic modulation of anxiety behaviors after intra-vHipp THC. Previous evidence has suggested that the anxiolytic and anxiogenic effects of low- vs high-dose CB1R agonists are independently modulated by activation of CB1Rs expressed on glutamatergic vs GABAergic neuronal populations (Rey et al., 2012). Furthermore, hippocampal CB1Rs are expressed at greater concentrations on GABAergic interneurons relative to glutamatergic neurons (Marsicano and Lutz, 1999; Nyíri et al., 2005; Takács et al., 2015) with CB1R activation preferentially targeting GABA interneurons (Pertwee, 2005; Roberto et al., 2010). These cell-specific differences could explain the differential anxiolytic-like vs anxiogeniclike effects of low-dose vs high dose THC, via saturation of CB1Rs localized on vHipp glutamatergic vs GABAergic neurons, respectively. While CBD had no effect alone, combined THC+CBD reversed THC-induced anxiogenesis, which was blocked by activation of vHipp pERK1-2 signaling, emphasizing the functional role of vHipp pERK1-2 inhibition in the anxiolytic properties of CBD. Thus, THC and CBD exert opposing control over anxietyrelated behavioral responses via differential regulation of vHipp ERK phosphorylation.

Hippocampal ERK activation is critically involved in fearrelated associative learning (Selcher et al., 2003; Huh et al., 2009; Ritov et al., 2014), and is necessary for reward-related and hippocampal-dependent memory formation (Berman et al., 1998; Besnard et al., 2013). In the current study, we found that intra-vHipp THC potentiated the rewarding properties of a normally subreward threshold conditioning dose of morphine and also amplified conditioned fear responses to context-dependent and context-independent cues. Both effects were blocked by MEK 1-2 inhibition or by CBD coadministration. These effects are consistent with changes in affective memory formation observed after acute vHipp CB1R, and GPR55 stimulation (Loureiro et al., 2015; Kramar et al., 2017), and support the hypothesis that THC dysregulates vHipp excitatory output leading to aberrant emotional sensory processing. Genetic variations of both the Akt and DA transporter genes are associated with dysregulation of DA signaling within the striatum and mesolimbic circuitry, as well as psychosis after acute, high dose THC exposure (Morgan et al., 2016; Colizzi et al., 2019). Intriguingly, we also observed a THC-specific generalization of fear responding to olfactory $\mathrm{CS}^{-}$ within context-independent fear testing, suggesting that intravHipp THC disrupts salience attribution toward affective sensory stimuli. The current design does not allow us to speculate as to whether this result is due to generalization of conditioned stimulus processing vs generalized negative affect. However, previous evidence suggests that $\mathrm{CBD}$ regulates fear-related memory processing by disrupting fear memory consolidation and enhancing fear extinction, both of which can result in a lasting reduction of learned fear (Norris et al., 2016; Lee et al., 2017), possibly underlying the mitigation of fear-responsivity after THC+CBD coadministration. Thus, similar to effects observed in anxiety-like behaviors, the combination of THC $+\mathrm{CBD}$ is capable of counteracting THC-induced affective memory changes by blocking THC-induced local ERK 1-2 activation.

Interestingly, THC, CBD, and their combination each increased natural, sucrose-related appetitive behaviors, suggesting that CBD-related mitigation of affective motivation may be selective for drug-related (vs natural) reward cues. Although CBD engendered few behavioral changes in the current study, changes in sucrose preference by systemic CBD administration is a well established phenomenon (Bisogno et al., 2001; Park et al., 2008).
The precise mechanism of action of CBD remains unknown, previous evidence largely excludes cannabinoid and vanilloid receptor contributions (Bisogno et al., 2001), and we have previously demonstrated the involvement of 5-HT1ARs in CBD-induced mitigation of emotional memory expression (Norris et al., 2016). Although CBD does not seem to have marked effects on any relevant physiological or symptomatic variables under normal circumstances (Martin-Santos et al., 2012), it appears to exert powerful modulation over cue-induced responsivity in associative learning tasks and in those with compromised neuropsychiatric statuses (Ren et al., 2009; Devinsky et al., 2014).

Through control of DA neuronal activity states, the vHipp relays affective-contextual information to downstream limbic regions. vHipp dysfunction may underlie DA dysregulation in schizophrenia, leading to impairments in salience attribution toward affective stimuli. For example, vHipp stimulation increases the number of spontaneously active VTA DA neurons and striatal DA influx (Legault et al., 2000; Floresco et al., 2001), and the psychotomimetic effects of THC are directly related to striatal DA and glutamate levels (Bhattacharyya et al., 2012; Fitoussi et al., 2018; Colizzi et al., 2019). Using in vivo single-cell electrophysiological recordings, we found that intra-vHipp THC significantly increased both frequency and bursting rates of VTA DA neurons. Interestingly, although CBD alone had no effect, coinfusion of $\mathrm{THC}+\mathrm{CBD}$, or THC + U0126 reversed THC-induced DA hyperactivity, demonstrating the direct involvement of vHipp ERK signaling in these effects.

Changes in VTA DA activity patterns from tonic to phasic burst firing are associated with phasic DA release in the nucleus accumbens, which encodes unexpected outcomes, such as prediction errors. Acute or neurodevelopmental THC exposure has been shown to induce a hyperactive mesolimbic DA state, both in terms of increased frequency and bursting in VTA DAergic neuronal populations (Renard et al., 2017a,b; Fitoussi et al., 2018). CB1Rs are predominantly expressed presynaptically on GABAergic axon terminals within the hippocampus and recent studies reveal that THC exhibits full agonist efficacy at these CB1Rs (Marsicano and Lutz, 1999; Nyíri et al., 2005; Laaris et al., 2010), possibly accounting for the hyperactive VTA DA endophenotype observed in our current study. They may also be relevant for interpreting the actions of the $\mathrm{THC}+\mathrm{CBD}$ combination; given that CBD increases endocannabinoid tone (Leishman et al., 2018), coadministered THC + CBD may indirectly antagonise the actions of THC on CB1Rs in the vHipp. Consistent with evidence that hippocampal ERK signaling facilitates generation of DAergic neuronal prediction errors (Huh et al., 2009), and that CBD normalizes VTA DA activity (Renard et al., 2016), coinfusion of CBD, or the MEK1-2 inhibitor U0126 each prevented the THCinduced increase in VTA DA phasic bursting. Although VTA DA vs non-DA neurons are well characterized and display canonical activity profiles in vivo, it has been previously demonstrated that a subset of tyrosine hydroxylase-positive VTA neurons display alternative physiological properties, and that no physiological property is both sensitive and selective for DA vs GABAergic neurons in the VTA (Margolis et al., 2006). Thus, it is important to recognize that the features used to characterize neuronal subpopulations in the current study are not ubiquitous.

In schizophrenia, loss of hippocampal GABAergic activity is hypothesized to disinhibit hippocampal outputs, inducing a hyperdopaminergic state (Grace, 2010). Reductions in PVexpressing interneurons are reliably observed in schizophrenia patients and are linked to NMDA receptor desensitization 
(Gonzalez-Burgos and Lewis, 2012). Similar aberrant volumetric deficits and physiological changes are observed in chronic cannabis users (Beale et al., 2018). Importantly, these local interneurons are necessary for the generation of normal $\gamma$ oscillations (Benes et al., 2007). In the VTA, LFPs are comprised of integrated input from several structures, including the hippocampus, nucleus accumbens and mPFC (Cembrowski et al., 2018). Considerable evidence demonstrates that dysregulated $\gamma$ oscillations contribute to schizophrenia-related perceptual and cognitive deficits (Baldeweg et al., 1998). $\gamma$ oscillations crucially subserve high-frequency oscillations (HFO), including $\varepsilon$, and frequently couple with slower frequencies, including $\beta$ (Buzsáki et al., 2012). HFOs, including $\varepsilon$, are evoked after hippocampal stimulation and coincide with excitotoxicity and seizure-like discharges (Fisher et al., 1992). Intriguingly, it has been suggested that $\beta$ and $\gamma$ frequency ranges may be particularly involved in long-range neural coordination (Kopell et al., 2000), and may underlie schizophrenia-related dysfunctional connectivity among cortical and subcortical networks. We observed increased oscillatory frequency in VTA beta, gamma, and $\varepsilon$ power after intra-vHipp THC. In contrast, although CBD alone had no effect alone, coinfusion of CBD, or the MEK1-2 inhibitor U0126, each counteracted these THC-induced effects on VTA oscillations suggesting functional involvement of vHipp ERK signaling in these oscillatory effects.

CB1R agonists dysregulate hippocampal and cortical beta and gamma magnitudes to a similar extent to that observed in schizophrenia (Morrison et al., 2011; Cass et al., 2014; Renard et al., 2017a,b). However, no studies to date have examined these effects directly in the VTA. Evidence suggests that CBD restores membrane excitability in PV- and CCK-expressing cells (Drysdale et al., 2006; Campos et al., 2013; Khan et al., 2018), suggesting that $\mathrm{CBD}$ coadministration may restore vHipp inhibitory control to counteract the neural effects of THC. That said, across several behavioral and electrophysiological measures, the effects of THC $+\mathrm{CBD}$ are different from those observed for vehicle alone, and in the opposite direction of THC. In other cases, CBD and THC each produce similar effects (Fig. 3e,f). It is important to note that the pharmacodynamic and pharmacokinetic mechanisms by which THC and CBD exert their effects are complicated, and become even more intricate when delivered in combination (Russo, 2011). Thus, it is not always possible to attribute the actions of the THC $+\mathrm{CBD}$ combination to one proposed molecular mechanism. Despite feasibility limitations and although outside of the scope of the present study, examining the actions of CBD + EPA, or THC + EPA may have aided interpretation across several measures. Given that interactions between hippocampal CB1R activation and ERK phosphorylation critically regulate DA neurotransmission and maintenance of normal oscillatory activity, the present findings provide further evidence for the contrasting effects of intra-vHipp THC/CBD on VTA oscillatory magnitudes.

In summary, the behavioral and neurophysiological disturbances elicited by intra-vHipp THC mimic a range of neuropsychiatric symptoms resembling core endophenotypes of schizophrenia. The present findings identify a common mechanism by which distinct phytocannabinoids may differentially modulate neuropsychiatric side effects of cannabis exposure through the bidirectional control of localized hippocampal ERK 1-2 phosphorylation states, mesolimbic DA activity levels and associated oscillatory wave patterns.

\section{References}

Baldeweg T, Spence S, Hirsch SR, Gruzelier J (1998) $\gamma$-band electroencephalographic oscillations in a patient with somatic hallucinations. Lancet 352:620-621.

Beale C, Broyd SJ, Chye Y, Suo C, Schira M, Galettis P, Martin JH, Yücel M, Solowij N (2018) Prolonged cannabidiol treatment effects on hippocampal subfield volumes in current cannabis users. Cannabis Cannabinoid Res 3:94-107.

Benes FM, Lim B, Matzilevich D, Walsh JP, Subburaju S, Minns M (2007) Regulation of the GABA cell phenotype in hippocampus of schizophrenics and bipolars. Proc Natl Acad Sci U S A 104:10164-10169.

Berman DE, Hazvi S, Rosenblum K, Seger R, Dudai Y (1998) Specific and differential activation of mitogen-activated protein kinase cascades by unfamiliar taste in the insular cortex of the behaving rat. J Neurosci 18:10037-10044.

Besnard A, Caboche J, Laroche S (2013) Recall and reconsolidation of contextual fear memory: differential control by ERK and Zif268 expression dosage. PLoS One 8:e72006.

Bhattacharyya S, Morrison PD, Fusar-Poli P, Martin-Santos R, Borgwardt S, Winton-Brown T, Nosarti C, O' Carroll CM, Seal M, Allen P, Mehta MA, Stone JM, Tunstall N, Giampietro V, Kapur S, Murray RM, Zuardi AW, Crippa JA, Atakan Z, McGuire PK (2010) Opposite effects of $\Delta-9$ tetrahydrocannabinol and cannabidiol on human brain function and psychopathology. Neuropsychopharmacology 35:764-774.

Bhattacharyya S, Crippa JA, Allen P, Martin-Santos R, Borgwardt S, FusarPoli P, Rubia K, Kambeitz J, O'carroll C, Seal ML, Giampietro V, Brammer M, Zuardi AW, Atakan Z, McGuire PK (2012) Induction of psychosis by $\delta 9$-tetrahydrocannabinol reflects modulation of prefrontal and striatal function during attentional salience processing. Arch Gen Psychiatry 69:27-36.

Bhattacharyya S, Falkenberg I, Martin-Santos R, Atakan Z, Crippa JA, Giampietro V, Brammer M, McGuire P (2015) Cannabinoid modulation of functional connectivity within regions processing attentional salience. Neuropsychopharmacology 40:1343-1352.

Bisogno T, Hanus L, De Petrocellis L, Tchilibon S, Ponde DE, Brandi I, Moriello AS, Davis JB, Mechoulam R, Di Marzo V (2001) Molecular targets for cannabidiol and its synthetic analogues: effect on vanilloid VR1 receptors and on the cellular uptake and enzymatic hydrolysis of anandamide. Br J Pharmacol 134:845-852.

Buzsáki G, Wang XJ (2012) Mechanisms of gamma oscillations. Annu Rev Neurosci 35:203-225.

Campos AC, Ortega Z, Palazuelos J, Fogaça MV, Aguiar DC, Díaz-Alonso J, Ortega-Gutiérrez S, Vázquez-Villa H, Moreira FA, Guzmán M, GalveRoperh I, Guimarães FS (2013) The anxiolytic effect of cannabidiol on chronically stressed mice depends on hippocampal neurogenesis: involvement of the endocannabinoid system. Int J Neuropsychopharmacol 16:1407-1419.

Cass DK, Flores-Barrera E, Thomases DR, Vital WF, Caballero A, Tseng KY (2014) CB1 cannabinoid receptor stimulation during adolescence impairs the maturation of GABA function in the adult rat prefrontal cortex. Mol Psychiatry 19:536-543.

Cembrowski MS, Wang L, Lemire AL, Copeland M, DiLisio SF, Clements J, Spruston N (2018) The subiculum is a patchwork of discrete subregions. eLife 7:e37701.

Colizzi M, Weltens N, McGuire P, Lythgoe D, Williams S, Van Oudenhove L, Bhattacharyya S (2019) Delta-9-tetrahydrocannabinol increases striatal glutamate levels in healthy individuals: implications for psychosis. Mol Psychiatry. Advance online publication. Retrieved February 15, 2019. doi: 10.1038/s41380-019-0374-8.

Derkinderen P, Valjent E, Toutant M, Corvol JC, Enslen H, Ledent C, Trzaskos J, Caboche J, Girault JA (2003) Regulation of extracellular signalregulated kinase by cannabinoids in hippocampus. J Neurosci 23:23712382.

Devinsky O, Cilio MR, Cross H, Fernandez-Ruiz J, French J, Hill C, Katz R, Di Marzo V, Jutras-Aswad D, Notcutt WG, Martinez-Orgado J, Robson PJ, Rohrback BG, Thiele E, Whalley B, Friedman D (2014) Cannabidiol: pharmacology and potential therapeutic role in epilepsy and other neuropsychiatric disorders. Epilepsia 55:791-802.

Di Forti M, Morgan C, Dazzan P, Pariante C, Mondelli V, Marques TR, Handley R, Luzi S, Russo M, Paparelli A, Butt A, Stilo SA, Wiffen B, Powell J, Murray RM (2009) High-potency cannabis and the risk of psychosis. Br J Psychiatry 195:488-491. 
Drysdale AJ, Ryan D, Pertwee RG, Platt B (2006) Cannabidiol-induced intracellular Ca2 + elevations in hippocampal cells. Neuropharmacology 50:621-631.

Duncia JV, Santella JB 3rd, Higley CA, Pitts WJ, Wityak J, Frietze WE, Rankin FW, Sun JH, Earl RA, Tabaka AC, Teleha CA, Blom KF, Favata MF, Manos EJ, Daulerio AJ, Stradley DA, Horiuchi K, Copeland RA, Scherle PA, Trzaskos JM, Magolda RL, Trainor GL, Wexler RR, Hobbs FW, Olson RE (1998) MEK inhibitors: the chemistry and biological activity of U0126, its analogs, and cyclization products. Bioorg Med Chem Lett $8: 2839-2844$.

Elbaz M, Nasser MW, Ravi J, Wani NA, Ahirwar DK, Zhao H, Oghumu S, Satoskar AR, Shilo K, Carson WE 3rd (2015) Modulation of the tumor microenvironment and inhibition of EGF/EGFR pathway: novel antitumor mechanisms of cannabidiol in breast cancer. Mol Oncol 9: 906-919.

Englund A, Morrison PD, Nottage J, Hague D, Kane F, Bonaccorso S, Stone JM, Reichenberg A, Brenneisen R, Holt D, Feilding A, Walker L, Murray RM, Kapur S (2013) Cannabidiol inhibits THC-elicited paranoid symptoms and hippocampal-dependent memory impairment. J Psychopharmacol 27:19-27.

Fisher RS, Webber WR, Lesser RP, Arroyo S, Uematsu S (1992) Highfrequency EEG activity at the start of seizures. J Clin Neurophysiol 9:441448.

Fitoussi A, Zunder J, Tan H, Laviolette SR (2018) Delta-9-tetrahydrocannabinol potentiates fear memory salience through functional modulation of mesolimbic dopaminergic activity states. Eur J Neurosci 47:1385-1400.

Floresco SB, Todd CL, Grace AA (2001) Glutamatergic afferents from the hippocampus to the nucleus accumbens regulate activity of ventral tegmental area dopamine neurons. J Neurosci 21:4915-4922.

Gomes FV, Issy AC, Ferreira FR, Viveros M-P, Del Bel EA, Guimarães FS (2015) Cannabidiol attenuates sensorimotor gating disruption and molecular changes induced by chronic antagonism of NMDA receptors in mice. Int J Neuropsychopharmacol 18.

Gonzalez-Burgos G, Lewis DA (2012) NMDA receptor hypofunction, parvalbumin-positive neurons, and cortical gamma oscillations in schizophrenia. Schizophr Bull 38:950-957.

Grace AA (2010) Ventral hippocampus, interneurons, and schizophrenia: a new understanding of the pathophysiology of schizophrenia and its implications for treatment and prevention. Current Directions in Psychological Science 19:232-237.

Grace AA, Bunney BS (1983) Intracellular and extracellular electrophysiology of nigral dopaminergic neurons. 1. Identification and characterization. Neuroscience 10:301-315.

Hájos N, Freund TF (2002) Distinct cannabinoid sensitive receptors regulate hippocampal excitation and inhibition. Chem Phys Lipids 121: $73-82$.

Huh KH, Guzman YF, Tronson NC, Guedea AL, Gao C, Radulovic J (2009) Hippocampal erk mechanisms linking prediction error to fear extinction: roles of shock expectancy and contextual aversive valence. Learn Mem 16:273-278.

Ivanov A, Pellegrino C, Rama S, Dumalska I, Salyha Y, Ben-Ari Y, Medina I (2006) Opposing role of synaptic and extrasynaptic NMDA receptors in regulation of the extracellular signal-regulated kinases (ERK) activity in cultured rat hippocampal neurons. J Physiol 572:789-798.

Khan AA, Shekh-Ahmad T, Khalil A, Walker MC, Ali AB (2018) Cannabidiol exerts antiepileptic effects by restoring hippocampal interneuron functions in a temporal lobe epilepsy model. Br J Pharmacol 175:20972115.

Kopell N, Ermentrout GB, Whittington MA, Traub RD (2000) Gamma rhythms and beta rhythms have different synchronization properties. Proc Natl Acad Sci U S A 97:1867-1872.

Kozlovsky N, Shanon-Weickert C, Tomaskovic-Crook E, Kleinman JE, Belmaker RH, Agam G (2004) Reduced GSK-3 mRNA levels in postmortem dorsolateral prefrontal cortex of schizophrenic patients. J Neural Transm 111:1583-1592.

Kramar C, Loureiro M, Renard J, Laviolette SR (2017) Palmitoylethanolamide modulates GPR55 receptor signaling in the ventral hippocampus to regulate mesolimbic dopamine activity, social interaction, and memory processing. Cannabis Cannabinoid Res 2:8-20.

Laaris N, Good CH, Lupica CR (2010) $\Delta$ 9-tetrahydrocannabinol is a full agonist at CB1 receptors on GABA neuron axon terminals in the hippocampus. Neuropharmacology 59:121-127.
Laviolette SR, Grace AA (2006) Cannabinoids potentiate emotional learning plasticity in neurons of the medial prefrontal cortex through basolateral amygdala inputs. J Neurosci 26:6458-6468.

Laviolette SR, van der Kooy D (2003) Blockade of mesolimbic dopamine transmission dramatically increases sensitivity to the rewarding effects of nicotine in the ventral tegmental area. Mol Psychiatry 8:50-59.

Lee JLC, Bertoglio LJ, Guimarães FS, Stevenson CW (2017) Cannabidiol regulation of emotion and emotional memory processing: relevance for treating anxiety-related and substance abuse disorders. Br J Pharmacol 174:3242-3256.

Legault M, Rompré PP, Wise RA (2000) Chemical stimulation of the ventral hippocampus elevates nucleus accumbens dopamine by activating dopaminergic neurons of the ventral tegmental area. J Neurosci 20:1635-1642.

Leishman E, Manchanda M, Thelen R, Miller S, Mackie K, Bradshaw HB (2018) Cannabidiol's upregulation of $\mathrm{N}$-acyl ethanolamines in the central nervous system requires $\mathrm{N}$-acyl phosphatidyl ethanolamine-specific phospholipase D. Cannabis Cannabinoid Res 3:228-241.

Leweke FM, Piomelli D, Pahlisch F, Muhl D, Gerth CW, Hoyer C, Klosterkötter J, Hellmich M, Koethe D (2012) Cannabidiol enhances anandamide signaling and alleviates psychotic symptoms of schizophrenia. Transl Psychiatry $2: e 94$.

Lintas A, Chi N, Lauzon NM, Bishop SF, Gholizadeh S, Sun N, Tan H, Laviolette SR (2011) Identification of a dopamine receptor-mediated opiate reward memory switch in the basolateral amygdala-nucleus accumbens circuit. J Neurosci 31:11172-11183.

Loureiro M, Renard J, Zunder J, Laviolette SR (2015) Hippocampal cannabinoid transmission modulates dopamine neuron activity: impact on rewarding memory formation and social interaction. Neuropsychopharmacology 40:1436-1447.

Loureiro M, Kramar C, Renard J, Rosen LG, Laviolette SR (2016) Cannabinoid transmission in the hippocampus activates nucleus accumbens neurons and modulates reward and aversion-related emotional salience. Biol Psychiatry 80:216-225.

Lyons D, de Jaeger X, Rosen LG, Ahmad T, Lauzon NM, Zunder J, Coolen LM, Rushlow W, Laviolette SR (2013) Opiate exposure and withdrawal induces a molecular memory switch in the basolateral amygdala between ERK1/2 and CaMKII $\alpha$-dependent signaling substrates. J Neurosci 33: 14693-14704.

Maher FO, Martin DS, Lynch MA (2004) Increased IL- $1 \beta$ in cortex of aged rats is accompanied by downregulation of ERK and PI-3 kinase. Neurobiol Aging 25:795-806.

Margolis EB, Lock H, Hjelmstad GO, Fields HL (2006) The ventral tegmental area revisited: is there an electrophysiological marker for dopaminergic neurons?. J Physiol 577:907-924.

Marsicano G, Lutz B (1999) Expression of the cannabinoid receptor CB1 in distinct neuronal subpopulations in the adult mouse forebrain. Eur J Neurosci 11:4213-4225.

Martin-Santos R, Crippa JA, Batalla A, Bhattacharyya S, Atakan Z, Borgwardt S, Allen P, Seal M, Langohr K, Farré M, Zuardi AW, McGuire PK (2012) Acute effects of a single, oral dose of d9-tetrahydrocannabinol (THC) and cannabidiol (CBD) administration in healthy volunteers. Curr Pharm Des 18, 4966-4979.

McPartland JM, Glass M, Pertwee RG (2007) Meta-analysis of cannabinoid ligand binding affinity and receptor distribution: interspecies differences. Br J Pharmacol 152:583-593.

Meyer-Lindenberg AS, Olsen RK, Kohn PD, Brown T, Egan MF, Weinberger DR, Berman KF (2005) Regionally specific disturbance of dorsolateral prefrontal-hippocampal functional connectivity in schizophrenia. Arch Gen Psychiatry 62:379-386.

Moreira FA, Guimarães FS (2005) Cannabidiol inhibits the hyperlocomotion induced by psychotomimetic drugs in mice. Eur J Pharmacol 512: $199-205$.

Morgan CJ, Freeman TP, Powell J, Curran HV (2016) AKT1 genotype moderates the acute psychotomimetic effects of naturalistically smoked cannabis in young cannabis smokers. Transl Psychiatry 6:e738.

Morrison PD, Nottage J, Stone JM, Bhattacharyya S, Tunstall N, Brenneisen R, Holt D, Wilson D, Sumich A, McGuire P, Murray RM, Kapur S, Ffytche DH (2011) Disruption of frontal theta coherence by $\Delta$ 9-tetrahydrocannabinol is associated with positive psychotic symptoms. Neuropsychopharmacology 36:827-836.

Norris C, Loureiro M, Kramar C, Zunder J, Renard J, Rushlow W, Laviolette SR (2016) Cannabidiol modulates fear memory formation through in- 
teractions with serotonergic transmission in the mesolimbic system. Neuropsychopharmacology 41:2839-2850.

Nyíri G, Cserép C, Szabadits E, Mackie K, Freund TF (2005) CB1 cannabinoid receptors are enriched in the perisynaptic annulus and on preterminal segments of hippocampal GABAergic axons. Neuroscience 136:811-822.

Ohl F (2003) Testing for anxiety. Clin Neurosci Res 3:233-238.

Palaniyappan L, Simmonite M, White TP, Liddle EB, Liddle PF (2013) Neural primacy of the salience processing system in schizophrenia. Neuron 79:814-828.

Park JB, Kwak HJ, Lee SH (2008) Role of hyaluronan in glioma invasion. Cell Adh Migr 2:202-207.

Paxinos G, Watson C (2007) The rat brain in stereotaxic coordinates. San Diego, CA: Academic.

Pertwee RG (2005) Inverse agonism and neutral antagonism at cannabinoid CB1 receptors. Life Sci 76:1307-1324.

Renard J, Loureiro M, Rosen LG, Zunder J, de Oliveira C, Schmid S, Rushlow WJ, Laviolette SR (2016) Cannabidiol counteracts amphetamineinduced neuronal and behavioral sensitization of the mesolimbic dopamine pathway through a novel mTOR/p70S6 kinase signaling pathway. J Neurosci 36:5160-5169.

Renard J, Rosen LG, Loureiro M, De Oliveira C, Schmid S, Rushlow WJ, Laviolette SR (2017a) Adolescent cannabinoid exposure induces a persistent sub-cortical hyper-dopaminergic state and associated molecular adaptations in the prefrontal cortex. Cereb Cortex 27:1297-1310.

Renard J, Szkudlarek HJ, Kramar CP, Jobson CEL, Moura K, Rushlow WJ, Laviolette SR (2017b) Adolescent THC exposure causes enduring prefrontal cortical disruption of GABAergic inhibition and dysregulation of sub-cortical dopamine function. Sci Rep 7:11420.

Ren Y, Whittard J, Higuera-Matas A, Morris CV, Hurd YL (2009) Cannabidiol, a nonpsychotropic component of cannabis, inhibits cue-induced heroin seeking and normalizes discrete mesolimbic neuronal disturbances. J Neurosci 29:14764-14769.

Rey AA, Purrio M, Viveros MP, Lutz B (2012) Biphasic effects of cannabinoids in anxiety responses: $\mathrm{CB} 1$ and GABA B receptors in the balance of GABAergic and glutamatergic neurotransmission. Neuropsychopharmacology 37:2624-2634.
Ritov G, Ardi Z, Richter-Levin G (2014) Differential activation of amygdala, dorsal and ventral hippocampus following an exposure to a reminder of underwater trauma. Front Behav Neurosci 8:18.

Roberto M, Cruz M, Bajo M, Siggins GR, Parsons LH, Schweitzer P (2010) The endocannabinoid system tonically regulates inhibitory transmission and depresses the effect of ethanol in central amygdala. Neuropsychopharmacology 35:1962-1972.

Russo EB (2011) Taming THC: potential cannabis synergy and phytocannabinoid-terpenoid entourage effects. Br J Pharmacol 163:1344-1364.

Salvati S, Natali F, Attorri L, Di Benedetto R, Leonardi F, Di Biase A, Ferri F, Fortuna S, Lorenzini P, Sanchez M, Ricceri L, Vitelli L (2008) Eicosapentaenoic acid stimulates the expression of myelin proteins in rat brain. J Neurosci Res 86:776-784.

Schubart CD, Sommer IE, van Gastel WA, Goetgebuer RL, Kahn RS, Boks MP (2011) Cannabis with high cannabidiol content is associated with fewer psychotic experiences. Schizophr Res 130:216-221.

Selcher JC, Weeber EJ, Christian J, Nekrasova T, Landreth GE, Sweatt JD (2003) A role for ERK MAP kinase in physiologic temporal integration in hippocampal area CA1. Learn Mem 10:26-39.

Solinas M, Massi P, Cinquina V, Valenti M, Bolognini D, Gariboldi M, Monti E, Rubino T, Parolaro D (2013) Cannabidiol, a non-psychoactive cannabinoid compound, inhibits proliferation and invasion in U87-MG and T98G glioma cells through a multitarget effect. PLoS One 8:e76918.

Szeszko PR, Goldberg E, Gunduz-Bruce H, Ashtari M, Robinson D, Malhotra AK, Lencz T, Bates J, Crandall DT, Kane JM, Bilder RM (2003) Smaller anterior hippocampal formation volume in antipsychotic-naive patients with first-episode schizophrenia. Am J Psychiatry 160:2190-2197.

Takács VT, Szőnyi A, Freund TF, Nyiri G, Gulyás AI (2015) Quantitative ultrastructural analysis of basket and axo-axonic cell terminals in the mouse hippocampus. Brain Struct Funct 220:919-940.

Yuan P, Zhou R, Wang Y, Li X, Li J, Chen G, Guitart X, Manji HK (2010) Altered levels of extracellular signal-regulated kinase signaling proteins in postmortem frontal cortex of individuals with mood disorders and schizophrenia. J Affect Disord 124:164-169.

Zuardi AW, Hallak JE, Dursun SM, Morais SL, Sanches RF, Musty RE, Crippa JA (2006) Cannabidiol monotherapy for treatment-resistant schizophrenia. J Psychopharmacol 20:683-686. 